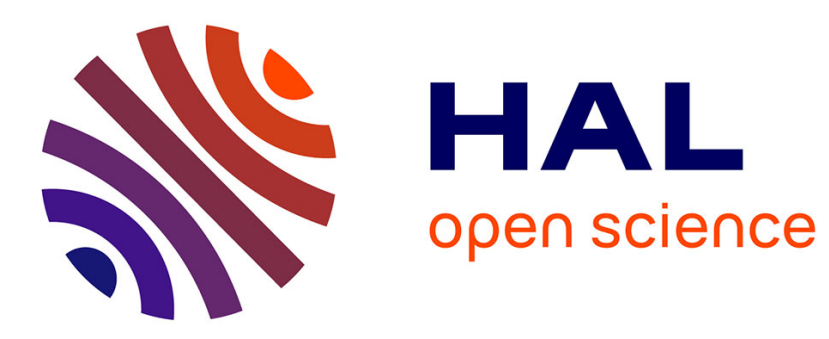

\title{
A Notion of Compliance Robustness in Topology Optimization
}

Samuel Amstutz, Marc Ciligot-Travain

\section{To cite this version:}

Samuel Amstutz, Marc Ciligot-Travain. A Notion of Compliance Robustness in Topology Optimization. ESAIM: Control, Optimisation and Calculus of Variations, 2016, 22 (1), pp.64-87. hal$00947108 \mathrm{v} 2$

\section{HAL Id: hal-00947108 \\ https://hal.science/hal-00947108v2}

Submitted on 8 May 2015

HAL is a multi-disciplinary open access archive for the deposit and dissemination of scientific research documents, whether they are published or not. The documents may come from teaching and research institutions in France or abroad, or from public or private research centers.
L'archive ouverte pluridisciplinaire $\mathbf{H A L}$, est destinée au dépôt et à la diffusion de documents scientifiques de niveau recherche, publiés ou non, émanant des établissements d'enseignement et de recherche français ou étrangers, des laboratoires publics ou privés. 


\title{
A NOTION OF COMPLIANCE ROBUSTNESS IN TOPOLOGY OPTIMIZATION
}

\author{
SAMUEL AMSTUTZ AND MARC CILIGOT-TRAVAIN
}

\begin{abstract}
The goal of this paper is twofold. On one hand, our work revisits the minimization of the robust compliance in shape optimization, with a more natural and more general approach than what has been done before. On the other hand, following a more recent viewpoint on robust optimization, we study the maximization of the so-called stability radius for a fixed maximal compliance. We provide theorical as well as numerical results.
\end{abstract}

\section{INTRODUCTION}

The compliance $C\left(\Omega, f_{\Omega}\right)$ of a linear elastic structure occupying a domain $\Omega$ and submitted to a load $f_{\Omega}$ is defined as the work done by the load, or equivalently as the stored elastic energy. Minimizing the compliance for a fixed load is a very standard shape optimization problem, for which a wide range of methods have been developed, see e.g. [1, 11] and the references therein. However, it often occurs that the load is not known exactly. In this work we suppose that it takes the form $f_{\Omega}^{N}+B_{\Omega} \xi, \xi \in r \mathbb{B}$, with $r>0, \mathbb{B}$ the closed unit ball of a Hibert space, $f_{\Omega}^{N}$ a nominal load and $B_{\Omega}$ a linear operator. The robust compliance (also called principal compliance) is then defined by

$$
j_{w c}(\Omega)=\sup _{\xi \in r \mathbb{B}} C\left(\Omega, f_{\Omega}^{N}+B_{\Omega} \xi\right) .
$$

The robust compliance may replace the compliance when the load is uncertain, so that minimizing the robust compliance is just minimizing the compliance 'in the worst case'. The way from compliance to robust compliance is just an illustration of the transition from optimization to robust optimization. The robust compliance has been first studied in [13], see also [14]. The worst case point of view has been applied to other criteria in $[2,8,23]$.

The goal of this paper is twofold. On one hand, our work revisits the paper by De Gournay et al [15] about the minimization of the robust compliance, with a more natural (and more general) approach. By definition, computing the robust compliance amounts to solving a quadratic optimization problem with a norm constraint in infinite dimension. In [15], the authors clearly announced that they renounced to follow this direct way because they did not see how to proceed, and used instead a formulation where the displacement field is chosen as main unknown. We show in the present article how to deal with the direct formulation.

On the other hand, following another and more recent viewpoint on robust optimization, we study the maximization of the so-called stability radius for a fixed

1991 Mathematics Subject Classification. 49Q10, 49M29, 74P05, 74P10, 74P15, 90C20.

Key words and phrases. Robustness, Stability radius, Compliance, Topological derivative, Topology Optimization. 
maximal compliance. This is actually the main purpose of this paper, and we shall enter a little more into details. Suppose that the compliance should not exceed $\alpha$. Then the stability radius relatively to the level-set $\left[C\left(\Omega, f_{\Omega}^{N}+B_{\Omega}.\right) \leq \alpha\right]$ is defined as

$$
\begin{aligned}
\sqrt{2 j_{s r}(\Omega)}=\sup \left\{r>0 \mid r \mathbb{B} \subset\left[C\left(\Omega, f_{\Omega}^{N}+B_{\Omega} .\right)\right.\right. & \leq \alpha]\} \\
& =\operatorname{dist}\left(0,\left[C\left(\Omega, f_{\Omega}^{N}+B_{\Omega} .\right)>\alpha\right]\right)
\end{aligned}
$$

Actually we shall rather consider the squared quantity $j_{s r}(\Omega)$, as it will be identified with the value of a quadratic programming problem. Then it is natural to look for the domain $\Omega$ which maximizes $j_{s r}(\Omega)$. In other words, we seek the shape which tolerates the greatest deviation from the nominal load, in the sense that the compliance remains below $\alpha$. This is a kind of robustness optimization. Actually, we will not necessarily find globally optimal shapes. It is more exact to say that we show how to improve the stability radius of a given shape until reaching, with respect to a certain class of perturbations, a locally optimal design.

The notion of stability radius appeared in robust control (see, e.g., [24, 25]), and has been developed in its full generality, but not from a very mathematical point of view, at the end of the 90's by Ben-Haim (see [9] and the references therein). Compared to the worst case approach, this one avoids fixing $r$ a priori, which is not necessarily easy and natural in some circumstances. We think that, in many situations, it is more natural to fix an upper bound $\alpha$ for the objective function, here the compliance. This amounts somehow to fixing some specifications.

The paper is organized as follows. In section 2, we describe the general mathematical setting of our problems. In section 3, we first show that the two robust criteria, namely $j_{w c}(\Omega)$ and $j_{s r}(\Omega)$, are the value functions of some quadratic programs with equality constraints. More precisely, the objective function of each subproblem is a quadratic functional and the equality constraint is associated with another quadratic functional. Such problems are known in the literature as trustregion subproblems, and have been extensively studied in the finite dimensional setting, see e.g. [16, 18, 22, 29, 17, 32, 35, 36, 37]. Here we prove the existence of critical loads (i.e. solutions of the subproblems) in arbitrary dimension, for both problems. Finally, using a strong duality argument for Lagrangian duality extending known results in finite dimension, we show the existence of a unique solution of the dual problem and give a complete description of the critical loads based on this solution. In section 4 , for both problems again, we give an expression of the Hadamard semiderivative of the two criteria relatively to the quadratic functionals depending on $\Omega$, based on the Lagrangian and the solution of the dual problem. Sections 5 through 7 specifically deal with the optimization problem with respect the shape $\Omega$. To keep concise and avoid repetitions, we concentrate on the maximization of the stability radius. Our procedure relies on the concept of topological derivative [21, 30,34], which evaluates the variation of the objective functional with respect to small topological perturbations. In section 5 , we deduce from section 4 the expression of the topological derivative of the stability radius. The optimization algorithm is described in section 6 , while section 7 reports on some numerical computations. 


\section{General Setting}

We denote by $\Omega$ the domain to be optimized, and by $\mathscr{E}$ the set of admissible domains. For each $\Omega \in \mathscr{E}$ we are given a reflexive Banach space $\mathcal{V}_{\Omega}$. We denote by $\|$.$\| the norm on \mathcal{V}_{\Omega}$ and by $\langle.,$.$\rangle the duality pairing between \mathcal{V}_{\Omega}^{\prime}$ and $\mathcal{V}_{\Omega}$, where $\mathcal{V}_{\Omega}^{\prime}$ stands for the continuous dual space of $\mathcal{V}_{\Omega}$. We also consider a continuous and self-adjoint positive definite isomorphism $A_{\Omega}$ from $\mathcal{V}_{\Omega}$ into $\mathcal{V}_{\Omega}^{\prime}$. We associate to each $f_{\Omega} \in \mathcal{V}_{\Omega}^{\prime}$ the vector $u_{\Omega, f_{\Omega}}=A_{\Omega}^{-1} f_{\Omega} \in \mathcal{V}_{\Omega}$ and the scalar

$$
C\left(\Omega, f_{\Omega}\right)=\frac{1}{2}\left\langle f_{\Omega}, u_{\Omega, f_{\Omega}}\right\rangle=\frac{1}{2}\left\langle f_{\Omega}, A_{\Omega}^{-1} f_{\Omega}\right\rangle
$$

Refering to the context of structural mechanics, we will subsequently call $f_{\Omega}$ the load, $u_{\Omega, f_{\Omega}}$ the displacement field, and $C\left(\Omega, f_{\Omega}\right)$ the compliance (actually the half compliance). We will consider a parameterized family of loads of the form

$$
f_{\Omega}=f_{\Omega}^{N}+B_{\Omega} \xi, \quad \xi \in \mathcal{H}
$$

where $f_{\Omega}^{N} \in \mathcal{V}_{\Omega}^{\prime}$ is a given nominal load, $\mathcal{H}$ is a fixed (i.e., independent of $\Omega$ ) separable Hilbert space and $B_{\Omega}: \mathcal{H} \rightarrow \mathcal{V}_{\Omega}^{\prime}$ is a linear, compact and injective operator. We set

$$
q(\Omega, \xi)=C\left(\Omega, f_{\Omega}^{N}+B_{\Omega} \xi\right)
$$

Hence we have, for $\xi \in \mathcal{H}$,

$$
q(\Omega, \xi)=\frac{1}{2}\left\langle Q_{\Omega} \xi, \xi\right\rangle+\left\langle b_{\Omega}, \xi\right\rangle+c_{\Omega}
$$

with

$$
Q_{\Omega}=B_{\Omega}^{*} A_{\Omega}^{-1} B_{\Omega}, \quad b_{\Omega}=B_{\Omega}^{*} A_{\Omega}^{-1} f_{\Omega}^{N}, \quad c_{\Omega}=\frac{1}{2}\left\langle f_{\Omega}^{N}, A_{\Omega}^{-1} f_{\Omega}^{N}\right\rangle .
$$

Note that $Q_{\Omega}: \mathcal{H} \rightarrow \mathcal{H}$ is a compact self-adjoint operator satisfying $Q_{\Omega} \geq 0$ and $Q_{\Omega} \neq 0$ (one supposes $\mathcal{H} \neq\{0\}$ ).

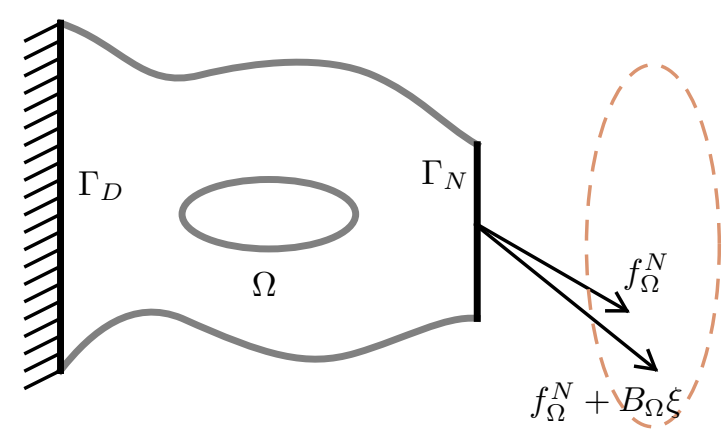

Before continuing with the abstract framework, let us give a typical concrete example. We consider the problem of compliance minimization for a structure submitted to an uncertain load. The structure is represented by a domain $\Omega \subset \mathbb{R}^{d}$ $(d=2$ or $d=3)$, whose boundary is split into three disjoint subsets $\Gamma, \Gamma_{D}$ and $\Gamma_{N}$ with meas $\left(\Gamma_{D}\right)>0$. Homogeneous Dirichlet boundary conditions are prescribed on $\Gamma_{D}$, and Neumann boundary conditions are prescribed on $\Gamma \cup \Gamma_{N}$, with zero force on $\Gamma$. We denote by $H_{D}^{1}(\Omega)^{d}$ the space of vector fields belonging to $H^{1}(\Omega)^{d}$ with 
vanishing trace on $\Gamma_{D}$. For a given load $f_{\Omega} \in\left(H_{D}^{1}(\Omega)^{d}\right)^{\prime}$ and a given displacement $u \in H_{D}^{1}(\Omega)^{d}$, the elastic energy is the quadratic functional in $u$ defined by

$$
E_{\Omega}\left(u, f_{\Omega}\right)=-\frac{1}{2} \int_{\Omega} H e(u): e(u)+\left\langle f_{\Omega}, u\right\rangle
$$

where $e(u)=\left(\nabla u+\nabla u^{T}\right) / 2$ is the strain tensor and $H$ is the fourth-order elasticity tensor (Hooke's tensor) such that $\mathrm{He}(u)$ is the stress tensor. The compliance is defined as

$$
C\left(\Omega, f_{\Omega}\right)=\max _{u \in H_{D}^{1}(\Omega)^{d}} E\left(u, f_{\Omega}\right)
$$

Therefore, setting $\mathcal{V}_{\Omega}=H_{D}^{1}(\Omega)^{d}$, the operator $A_{\Omega}: \mathcal{V}_{\Omega} \rightarrow \mathcal{V}_{\Omega}^{\prime}$ is defined by

$$
\left\langle A_{\Omega} u, v\right\rangle=\int_{\Omega} H e(u): e(v)
$$

and the compliance admits the expression

$$
C\left(\Omega, f_{\Omega}\right)=\frac{1}{2}\left\langle f_{\Omega}, A_{\Omega}^{-1} f_{\Omega}\right\rangle
$$

We choose $\mathcal{H}$ as a closed subspace of $L^{2}\left(\Gamma_{N}\right)^{d}$, and we define $B_{\Omega}: \mathcal{H} \rightarrow \mathcal{V}_{\Omega}^{\prime}$ by

$$
B_{\Omega} \xi:\left.u \in \mathcal{V}_{\Omega} \mapsto \int_{\Gamma_{N}} \xi \cdot u\right|_{\Gamma_{N}} .
$$

The compactness of $B_{\Omega}$ is due to the compactness of the Sobolev embedding $L^{2}\left(\Gamma_{N}\right) \rightarrow H^{-1 / 2}\left(\Gamma_{N}\right)$. A very standard problem in optimal design consists in minimizing the compliance with a fixed load $f_{\Omega}^{N}$, i.e.,

$$
\text { Minimize } q(\Omega, 0)=C\left(\Omega, f_{\Omega}^{N}\right), \quad \Omega \in \mathscr{E},
$$

where the set $\mathscr{E}$ can include constraints. One speaks of robust compliance minimization when, at the same time, perturbations of the load are considered.

Let us now come back to the general case. In this paper we will investigate two notions of robustness and the associated optimization problems.

(1) Stability radius as compliance robustness. Given a threshold $\alpha>$ $C\left(\Omega, f_{\Omega}^{N}\right)=c_{\Omega}$, the functional $j_{s r}$ is defined by

$$
j_{s r}(\Omega)=\frac{1}{2} \operatorname{dist}(0,[q(\Omega, .)>\alpha])^{2}=\inf _{q(\Omega, \xi)>\alpha} \frac{1}{2}\|\xi\|^{2}=\inf _{C\left(\Omega, f_{\Omega}^{N}+B_{\Omega} \xi\right)>\alpha} \frac{1}{2}\|\xi\|^{2} .
$$

Increasing this value amounts to increasing the distance to unfeasability, where unfeasability means that the compliance is greater than $\alpha$. This leads to considering the optimization problem:

$$
\text { Maximize } j_{s r}(\Omega), \quad \Omega \in \mathscr{E},
$$

hence one speaks of robustness maximization.

(2) Robust compliance in the worst case sense. Given a radius $r>0$, the worst case compliance is defined by

$$
j_{w c}(\Omega)=\sup _{\|\xi\| \leq r} q(\Omega, \xi)=\sup _{\|\xi\| \leq r} C\left(\Omega, f_{\Omega}^{N}+B_{\Omega} \xi\right) .
$$

This is the maximal compliance obtained for a given family of loads. One naturally wants to minimize this quantity, leading to the so-called worst case compliance minimization problem:

$$
\text { Minimize } j_{w c}(\Omega), \quad \Omega \in \mathscr{E} \text {. }
$$


In fact it is easily checked that the inequalities in (2.2) and (2.3) can be replaced by equalities, i.e. we have

$$
\begin{gathered}
j_{s r}(\Omega)=\frac{1}{2} \operatorname{dist}(0,[q(\Omega, .)=\alpha])^{2}=\inf _{q(\Omega, \xi)=\alpha} \frac{1}{2}\|\xi\|^{2}=\inf _{C\left(\Omega, f_{\Omega}^{N}+B_{\Omega} \xi\right)=\alpha} \frac{1}{2}\|\xi\|^{2} . \\
j_{w c}(\Omega)=\sup _{\|\xi\|=r} q(\Omega, \xi)=\sup _{\|\xi\|=r} C\left(\Omega, f_{\Omega}^{N}+B_{\Omega} \xi\right) .
\end{gathered}
$$

\section{EXPRESSION OF THE ROBUST CRITERIA}

In all this section the domain $\Omega$ is fixed. Hence, for notational simplicity, we drop the subscript $\Omega$, denoting $Q_{\Omega}, b_{\Omega}, c_{\Omega}$ by $Q, b, c$.

Actually, the framework of this section does not require that $Q, b, c$ be necessarily defined by (2.1). It is sufficient to assume that $Q: \mathcal{H} \rightarrow \mathcal{H}$ is a compact, self-adjoint operator with $Q \geq 0, Q \neq 0, b \in \mathcal{H}$, and $\alpha, r, c \in \mathbb{R}$ satisfy $c<\alpha$ and $r>0$.

We shall give practical procedures to compute the values of $j_{s r}(\Omega)$ and $j_{w c}(\Omega)$.

The two optimization problems appearing in (2.4) and (2.5) can be formulated in the form

$$
\text { Minimize } q_{1}(\xi), \quad \text { subject to } q_{2}(\xi)=0, \quad \xi \in \mathcal{H},
$$

with two quadratic functionals $q_{1}$ and $q_{2}$ written as

$$
q_{1}(\xi)=\frac{1}{2}\left\langle Q_{1} \xi, \xi\right\rangle+\left\langle b_{1}, \xi\right\rangle+c_{1}, \quad q_{2}(\xi)=\frac{1}{2}\left\langle Q_{2} \xi, \xi\right\rangle+\left\langle b_{2}, \xi\right\rangle+c_{2}
$$

In all this section, these quantities are defined as follows, where $I$ is the identity of $\mathcal{H}$.

(1) Compliance robustness :

$$
q_{1}(\xi)=\frac{1}{2}\|\xi\|^{2}, \quad q_{2}(\xi)=q(\Omega, \xi)-\alpha
$$

i.e.,

$$
\begin{array}{lll}
Q_{1}=I, & b_{1}=0, & c_{1}=0, \\
Q_{2}=Q, & b_{2}=b, & c_{2}=c-\alpha .
\end{array}
$$

(2) Worst case robust compliance :

$$
\begin{aligned}
& q_{1}(\xi)=-q(\Omega, \xi), \quad q_{2}(\xi)=\frac{1}{2}\|\xi\|^{2}-\frac{1}{2} r^{2}, \\
& Q_{1}=-Q, \\
& b_{1}=-b \text {, } \\
& c_{1}=-c \text {, } \\
& Q_{2}=I \text {, } \\
& b_{2}=0 \text {, } \\
& c_{2}=-r^{2} / 2 \text {. }
\end{aligned}
$$

Problems of form $(\mathscr{P})$ are known in the literature as trust region problems (or subproblems). They have been extensively studied, but almost always in the case of a finite dimensional space $\mathcal{H}$, see e.g. $[16,18,22,29,17,32,35,36,37]$. 


\subsection{Existence of critical loads.}

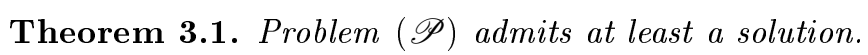

Proof. Consider first the case (3.1). In this case, $Q_{2}$ is compact with $Q_{2}=Q \geq 0$, $Q_{2} \neq 0$ and $c_{2}=c-\alpha<0$. Hence, $q_{2}(0)<0$, there exists $\xi_{0}$ such that $q_{2}\left(\xi_{0}\right)>0$ so $\left[q_{2}=0\right] \neq \emptyset$. Let $\left(\xi_{n}\right)$ be a minimizing sequence. For any $\beta>0,\left[q_{1} \leq \beta\right]$ is a closed ball. Thus, up to a subsequence, there exists $\xi \in \mathcal{H}$ such that $\xi_{n} \rightarrow$ $\xi$. By compactness of $Q_{2}$, we have $q_{2}(\xi)=\lim q_{2}\left(\xi_{n}\right)=0$. Since $q_{1}$ is convex and continuous, it is weakly lower-semicontinuous, thus $q_{1}(\xi) \leq \liminf q_{1}\left(\xi_{n}\right)=$ $\inf _{\left[q_{2}=0\right]} q_{1}$.

Let us turn to the second case (3.2). Recall that, in this case, $Q_{1}$ is compact, negative semi-definite. Let $\left(\xi_{n}\right)$ be a minimizing sequence of the problem $\inf _{\left[q_{2} \leq 0\right]} q_{1}$. $\left[q_{2} \leq 0\right]$ is a closed ball. Thus, up to a subsequence, there exists $\xi \in \mathcal{H}$ such that $\xi_{n} \rightarrow \xi$. By compactness of $Q_{1}$, we have $q_{1}(\xi)=\lim q_{1}\left(\xi_{n}\right)=\inf _{\left[q_{2} \leq 0\right]} q_{1}$. As $q_{2}$ is convex and continuous, it is weakly lower-semicontinuous, thus $q_{2}(\xi) \leq$ $\liminf q_{2}\left(\xi_{n}\right) \leq 0$. Therefore, the infimum $\inf _{\left[q_{2} \leq 0\right]} q_{1}$ is attained at some points of $\left[q_{2} \leq 0\right]$. Finally, as $q_{1}$ is concave and $\left[q_{2} \leq 0\right]$ is convex, at least one of these points can be found in $\left[q_{2}=0\right]$.

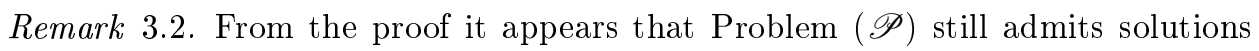
if $\left(Q_{2}, b_{2}, c_{2}\right)$, with $Q_{2}: \mathcal{H} \rightarrow \mathcal{H}$ self-adjoint, compact, is sufficiently closed to $\left(\bar{Q}_{2}, \bar{b}_{2}, \bar{c}_{2}\right)$ with $\bar{Q}_{2}: \mathcal{H} \rightarrow \mathcal{H}$ self-adjoint, compact, $\bar{Q}_{2} \geq 0, \bar{Q}_{2} \neq 0$ and $\bar{c}_{2}<\alpha$.

3.2. Dual formulation: general framework. For all $(\xi, \mu) \in \mathcal{H} \times \mathbb{R}$ we associate to Problem $(\mathscr{P})$ the Lagrangian

$$
L(\xi, \mu)=q_{1}(\xi)+\mu q_{2}(\xi)=\frac{1}{2}\left\langle\left(Q_{1}+\mu Q_{2}\right) \xi, \xi\right\rangle+\left\langle b_{1}+\mu b_{2}, \xi\right\rangle+c_{1}+\mu c_{2} .
$$

The dual criterion is

$$
g(\mu)=\inf _{\xi \in \mathcal{H}} L(\xi, \mu)
$$

For any self-adjoint linear continuous operator $T: \mathcal{H} \rightarrow \mathcal{H}$, we have

$$
\mathcal{H}=\operatorname{ker} T \stackrel{\perp}{\oplus} \operatorname{cl}(\operatorname{im} T)
$$

hence the restriction $\left.T\right|_{\operatorname{cl}(\operatorname{im} T)}: \operatorname{cl}(\operatorname{im} T) \rightarrow \operatorname{im} T$ is a bijection. We denote by $T^{\dagger}:=\left.T\right|_{\operatorname{cl}(\mathrm{im} T)} ^{-1}: \operatorname{im} T \rightarrow \operatorname{cl}(\operatorname{im} T)$ the inverse operator, which, by virtue of the open mapping theorem, is continuous as soon as $\operatorname{im} T$ is closed. For all $\mu \in \mathbb{R}$ such that $b_{1}+\mu b_{2} \in \operatorname{im}\left(Q_{1}+\mu Q_{2}\right)$ we set

$$
\psi(\mu)=-\frac{1}{2}\left\langle\left(Q_{1}+\mu Q_{2}\right)^{\dagger}\left(b_{1}+\mu b_{2}\right), b_{1}+\mu b_{2}\right\rangle+c_{1}+\mu c_{2} .
$$

Lemma 3.3. The dual criterion is expressed by

$$
g(\mu)= \begin{cases}-\infty & \text { if } Q_{1}+\mu Q_{2} \geq 0, \\ -\infty & \text { if } Q_{1}+\mu Q_{2} \geq 0 \text { and } b_{1}+\mu b_{2} \notin \operatorname{im}\left(Q_{1}+\mu Q_{2}\right), \\ \psi(\mu) & \text { if } Q_{1}+\mu Q_{2} \geq 0 \text { and } b_{1}+\mu b_{2} \in \operatorname{im}\left(Q_{1}+\mu Q_{2}\right) .\end{cases}
$$

Proof. We first note that the condition

$$
\forall \mu \in \mathbb{R}, \quad Q_{1}+\mu Q_{2} \geq 0 \Longrightarrow \operatorname{im}\left(Q_{1}+\mu Q_{2}\right) \text { is closed }
$$

is fulfilled in the two cases under study. Indeed, in the first case, we have $Q_{1}+\mu Q_{2}=$ $I+\mu Q$, whose image is always closed since $Q$ is compact. In the second case we 
have $Q_{1}+\mu Q_{2}=-Q+\mu I$, and $Q_{1}+\mu Q_{2} \geq 0$ implies $\mu>0$, whereby we conclude as before. For simplicity, we set $Q_{\mu}=Q_{1}+\mu Q_{2}, b_{\mu}=b_{1}+\mu b_{2}, c_{\mu}=c_{1}+\mu c_{2}$, so that

$$
g(\mu)=\inf _{\xi \in \mathcal{H}} q_{\mu}(\xi):=\frac{1}{2}\left\langle Q_{\mu} \xi, \xi\right\rangle+\left\langle b_{\mu}, \xi\right\rangle+c_{\mu} .
$$

If $Q_{\mu} \geq 0$, it is clear that $g(\mu)=-\infty$. Therefore we assume now that $Q_{\mu} \geq 0$. By (3.3), $\operatorname{im} Q_{\mu}$ is closed, thus

$$
\mathcal{H}=\operatorname{ker} Q_{\mu} \stackrel{\perp}{\oplus} \operatorname{im} Q_{\mu} .
$$

For all $\xi \in \mathcal{H}$, we make the decomposition $\xi=\xi_{1}+\xi_{2}$, with $\xi_{1} \in \operatorname{ker} Q_{\mu}$ and $\xi_{2} \in \operatorname{im} Q_{\mu}$. We get

$$
q_{\mu}(\xi)=q_{\mu}\left(\xi_{2}\right)+\left\langle b_{\mu}, \xi_{1}\right\rangle
$$

Two cases can arise.

(1) If $b_{\mu} \notin \operatorname{im} Q_{\mu}$, choosing $\xi=t \tilde{b}_{\mu}$ with $t \in \mathbb{R}$ and $\tilde{b}_{\mu}$ the orthogonal projection of $b_{\mu}$ onto ker $Q_{\mu}$, we obtain

$$
q_{\mu}(\xi)=t\left\|b_{\mu}\right\|^{2} .
$$

Letting $t$ go to $-\infty$ yields $g(\mu)=-\infty$

(2) If $b_{\mu} \in \operatorname{im} Q_{\mu}$, we have $q_{\mu}(\xi)=q_{\mu}\left(\xi_{2}\right)$ for all $\xi \in \mathcal{H}$, and

$$
g(\mu)=\inf _{\xi_{2} \in \operatorname{im} Q_{\mu}} q_{\mu}\left(\xi_{2}\right):=\frac{1}{2}\left\langle Q_{\mu} \xi_{2}, \xi_{2}\right\rangle+\left\langle b_{\mu}, \xi_{2}\right\rangle+c_{\mu} .
$$

The unique minimizer of this quadratic problem is $\xi_{2}=-Q_{\mu}^{\dagger} b_{\mu}$, and the value of the minimum is $-\frac{1}{2}\left\langle Q_{\mu}^{\dagger} b_{\mu}, b_{\mu}\right\rangle+c_{\mu}$, i.e., $\psi(\mu)$.

The dual problem is

(D) Maximize $\psi(\mu)$ subject to $\left\{\begin{array}{l}Q_{1}+\mu Q_{2} \geq 0, \\ b_{1}+\mu b_{2} \in \operatorname{im}\left(Q_{1}+\mu Q_{2}\right),\end{array} \quad \mu \in \mathbb{R}\right.$.

The following result is an adaptation of Theorem 2.1 of [37]. Due to its importance in the sequel, we nevertheless give a proof.

Theorem 3.4. Let $\bar{\xi} \in \mathcal{H}$. The following statements are equivalent:

(1) $\bar{\xi}$ is a (global) minimizer of $(\mathscr{P})$;

(2) $q_{2}(\bar{\xi})=0$ and there exists $\bar{\mu} \in \mathbb{R}$ such that

$$
\begin{gathered}
\partial_{\xi} L(\bar{\xi}, \bar{\mu})=\left(Q_{1}+\bar{\mu} Q_{2}\right) \bar{\xi}+\left(b_{1}+\bar{\mu} b_{2}\right)=0, \\
\partial_{\xi \xi}^{2} L(\bar{\xi}, \bar{\mu})=Q_{1}+\bar{\mu} Q_{2} \geq 0
\end{gathered}
$$

(3) there exists $\bar{\mu} \in \mathbb{R}$ such that

$$
\bar{\xi} \in \operatorname{argmin} L(., \bar{\mu}) \cap\left[q_{2}=0\right] .
$$

Proof. We shall prove the implications $(1) \Rightarrow(2) \Rightarrow(3) \Rightarrow(1)$.

First step. Let us assume that $\bar{\xi}$ is a minimizer of $(\mathscr{P})$. As the constraint is scalar, the classical constraint qualification reduces to

$$
\nabla q_{2}(\bar{\xi})=Q_{2} \bar{\xi}+b_{2} \neq 0 .
$$

In case (3.1), $Q_{2} \bar{\xi}+b_{2}=Q \bar{\xi}+b \neq 0$ since otherwise the constraint $q_{2}(\xi)=0$ would yield $\langle Q \bar{\xi}, \bar{\xi}\rangle=2(c-\alpha)<0$. In case $(3.2), Q_{2} \bar{\xi}+b_{2}=\bar{\xi} \neq 0$ since $\|\bar{\xi}\|=r>0$. 
Therefore, by the first order necessary optimality condition (see e.g. [27] 1.1.1, Theorem 1), there exists $\bar{\mu} \in \mathbb{R}$ such that

$$
\partial_{\xi} L(\bar{\xi}, \bar{\mu})=0 .
$$

The second order necessary optimality condition reads (see e.g. [27] 7.2.1, Corollary of Theorem 1)

$$
\begin{aligned}
& \left\langle\partial_{\xi \xi}^{2} L(\bar{\xi}, \bar{\mu}) \zeta, \zeta\right\rangle \geq 0 \quad \forall \zeta \in T_{\bar{\xi}}, \\
& T_{\bar{\xi}}=\left\{\zeta \in \mathcal{H},\left\langle Q_{2} \bar{\xi}+b_{2}, \zeta\right\rangle=0\right\} .
\end{aligned}
$$

Now, suppose that $\zeta \notin T_{\bar{\xi}}$. We assume first that $Q_{2} \zeta \neq 0$. Since $Q_{2} \geq 0$, this entails $\left\langle Q_{2} \zeta, \zeta\right\rangle>0$. Set

$$
\bar{t}=-2 \frac{\left\langle Q_{2} \bar{\xi}+b_{2}, \zeta\right\rangle}{\left\langle Q_{2} \zeta, \zeta\right\rangle} \neq 0, \quad \xi=\bar{\xi}+\bar{t} \zeta .
$$

After calculation we find that $q_{2}(\xi)=q_{2}(\bar{\xi})=0$. This implies

$$
L(\xi, \bar{\mu})-L(\bar{\xi}, \bar{\mu})=q_{1}(\xi)-q_{1}(\bar{\xi}) \geq 0 .
$$

Yet we have

$$
L(\xi, \bar{\mu})-L(\bar{\xi}, \bar{\mu})=\bar{t}\left\langle\left(Q_{1}+\bar{\mu} Q_{2}\right) \bar{\xi}+b_{1}+\bar{\mu} b_{2}, \zeta\right\rangle+\frac{\bar{t}^{2}}{2}\left\langle\left(Q_{1}+\bar{\mu} Q_{2}\right) \zeta, \zeta\right\rangle .
$$

Combining (3.4), (3.9), (3.10) and $\bar{t} \neq 0$, we derive that $\left\langle\left(Q_{1}+\bar{\mu} Q_{2}\right) \zeta, \zeta\right\rangle \geq 0$. Assume now that $Q_{2} \zeta=0$. As $Q_{2} \neq 0$, we choose some $\xi_{0} \in \mathcal{H}$ such that $Q_{2} \xi_{0} \neq 0$. Let $\left(t_{n}\right)$ be an arbitrary sequence of positive real numbers such that $t_{n} \rightarrow 0$ as $n \rightarrow \infty$. We set $\zeta_{n}=\zeta+t_{n} \xi_{0}$. For all $n$ we have $Q_{2} \zeta_{n}=t_{n} Q_{2} \xi_{0} \neq 0$, hence $\left\langle\left(Q_{1}+\bar{\mu} Q_{2}\right) \zeta_{n}, \zeta_{n}\right\rangle \geq 0$. Passing to the limit yields $\left\langle\left(Q_{1}+\bar{\mu} Q_{2}\right) \zeta, \zeta\right\rangle \geq 0$. We have proved that $Q_{1}+\bar{\mu} Q_{2} \geq 0$. In other words, the second order optimality condition (3.8) holds for any $\zeta$, which is a typical fact in quadratic programming.

Second step. Since the function $\xi \mapsto L(\xi, \bar{\mu})$ is quadratic, a Taylor expansion immediately shows that the conditions (3.4) and (3.5) imply $\bar{\xi} \in \operatorname{argmin} L(., \bar{\mu})$.

Third step. We remark that, for all $\mu \in \mathbb{R}$, it holds

$$
\left[L(., \mu)=q_{1}+\delta_{\left[q_{2}=0\right]}\right]=\left[q_{2}=0\right],
$$

with $\delta_{C}$ the indicator function of the set $C$, i.e., $\delta_{C}(x)=0$ if $x \in C$ and $\delta_{C}(x)=+\infty$ if $x \notin C$. By (A.2) in the appendix, we have $\bar{\xi} \in \operatorname{argmin} L(., \bar{\mu}) \cap\left[q_{2}=0\right] \subset$ $\operatorname{argmin} q_{1}+\delta_{\left[q_{2}=0\right]}$.

Remark 3.5. One can also deduce the implication $(1 \Rightarrow 3)$ of Theorem 3.4 from the following version of the S-lemma (see [19] for the original version in Russian).

Theorem 3.6 (S-lemma, [20], Theorem 2.1). Let $s_{i}: \mathcal{H} \rightarrow \mathbb{R}, i=1,2$, be two quadratic functionals of the form $s_{i}: \xi \rightarrow \frac{1}{2}\left\langle S_{i} \xi, \xi\right\rangle+\left\langle d_{i}, \xi\right\rangle+e_{i}, S_{i}: \mathcal{H} \rightarrow \mathcal{H}$ continuous and self-adjoint, $d_{i} \in \mathcal{H}$ and $e_{i} \in \mathbb{R}$. Suppose that $s_{2}$ is nonlinear, that there exist $\xi_{+}, \xi_{-} \in \mathcal{H}$ such that $s_{2}\left(\xi_{+}\right)>0, s_{2}\left(\xi_{-}\right)<0$ and

$$
\forall \xi \in \mathcal{H}, \quad s_{2}(\xi)=0 \Longrightarrow s_{1}(\xi) \geq 0 .
$$

Then there exists $\lambda \in \mathbb{R}$ such that

$$
\forall \xi \in \mathcal{H}, \quad s_{1}(\xi)-\lambda s_{2}(\xi) \geq 0 .
$$

In fact, one sets $s_{1}=q_{1}-q_{1}(\bar{\xi}), s_{2}=q_{2}, s_{2}$ is nonlinear because $Q_{2} \neq 0$, and there exist $\xi_{+}, \xi_{-} \in \mathcal{H}$ such that $s_{2}\left(\xi_{+}\right)>0, s_{2}\left(\xi_{-}\right)<0$ due to $(3.7)$ and $q_{2}(\bar{\xi})=0$. Then $(1 \Rightarrow 3)$ of Theorem 3.4 results from the S-lemma 3.6 . 
The following Theorem is a consequence of Theorem 3.4 together with general results on duality (cf. appendix A).

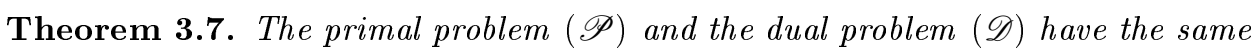
optimal values. In addition, $(\mathscr{D})$ admits solutions, and, if $\bar{\mu}$ is one of these solutions, we have

$$
\operatorname{argmin}(\mathscr{P})=\operatorname{argmin} L(., \bar{\mu}) \cap\left[q_{2}=0\right] .
$$

Proof. In view of Theorem 3.1, Theorem 3.4 and (3.11), one uses successively (A.5), (A.3) and (A.6).

3.3. Expression of the critical loads for the compliance robustness. In this case, with the notations $(2.1)$, the primal problem $(\mathscr{P})$ and the dual problem $(\mathscr{D})$ read, respectively,

(3.12) Minimize $\frac{1}{2}\|\xi\|^{2} \quad$ subject to $q(\xi):=\frac{1}{2}\langle Q \xi, \xi\rangle+\langle b, \xi\rangle+c=\alpha, \quad \xi \in \mathcal{H}$,

$$
\begin{aligned}
\text { Maximize } \psi_{s r}(\mu)=-\frac{1}{2}\left\langle(I+\mu Q)^{\dagger}(\mu b), \mu b\right\rangle+\mu(c-\alpha) \\
\text { subject to }\left\{\begin{array}{l}
I+\mu Q \geq 0, \\
\mu b \in \operatorname{im}(I+\mu Q),
\end{array} \quad \mu \in \mathbb{R} .\right.
\end{aligned}
$$

We denote by $\lambda_{\max }$ the largest eigenvalue of $Q$. The following theorem refines Theorem 3.7 and uses the same terminology as [37].

Theorem 3.8. The primal problem (3.12) and the dual problem (3.13) have the same optimal values. The dual problem (3.13) admits a unique solution $\bar{\mu} \in \mathbb{R}$, which can be computed in the following way.

- Easy case: $b \notin \operatorname{ker}\left(Q-\lambda_{\max } I\right)^{\perp}$. Then $\bar{\mu}$ is the unique solution in ]$1 / \lambda_{\max }, 0[$ of the equation

$$
q\left(-\mu(I+\mu Q)^{-1} b\right)=\alpha .
$$

- Hard case $I: b \in \operatorname{ker}\left(Q-\lambda_{\max } I\right)^{\perp}$ and

$$
q\left(\left(\lambda_{\max } I-Q\right)^{\dagger} b\right)>\alpha .
$$

Then $\bar{\mu}$ is also the unique solution in $]-1 / \lambda_{\max }, 0[$ of (3.14).

- Hard case II: $b \in \operatorname{ker}\left(Q-\lambda_{\max } I\right)^{\perp}$ and $q\left(\left(\lambda_{\max } I-Q\right)^{\dagger} b\right) \leq \alpha$. Then $\bar{\mu}=-1 / \lambda_{\max }$.

The set $\Xi$ of solutions of the primal problem (3.12) is given by the following expressions.

- Easy case and Hard case I. There is a unique critical load given by

$$
\Xi=\left\{-\bar{\mu}(I+\bar{\mu} Q)^{-1} b\right\} .
$$

- Hard case II. The set of critical loads is

$$
\Xi=\left[\left\{\left(\lambda_{\max } I-Q\right)^{\dagger} b\right\}+\operatorname{ker}\left(Q-\lambda_{\max } I\right)\right] \cap[q=\alpha] .
$$

Proof. Let us reformulate the constraints of the dual problem (3.13). The first one is equivalent to $\mu \geq-1 / \lambda_{\max }$, and we have by the classical theory of compact 
perturbations of the identity $\operatorname{im}(I+\mu Q)=\operatorname{ker}(I+\mu Q)^{\perp}=\mathcal{H}$ as soon as $\mu>$ $-1 / \lambda_{\max }$. Therefore we have

$$
\left\{\begin{array}{l}
I+\mu Q \geq 0 \\
\mu b \in \operatorname{im}(I+\mu Q)
\end{array}\right\} \Longleftrightarrow\left\{\begin{array}{l}
\mu>-1 / \lambda_{\max } \text { if } b \notin \operatorname{ker}\left(Q-\lambda_{\max } I\right)^{\perp} \\
\mu \geq-1 / \lambda_{\max } \text { if } b \in \operatorname{ker}\left(Q-\lambda_{\max } I\right)^{\perp}
\end{array}\right\} .
$$

If $-1 / \lambda_{\max }<\mu$ we have

$$
\psi_{s r}(\mu)=-\frac{\mu^{2}}{2}\left\langle(I+\mu Q)^{-1} b, b\right\rangle+\mu(c-\alpha) .
$$

Differentiating entails

$$
\begin{aligned}
\psi_{s r}^{\prime}(\mu) & =-\mu\left\langle(I+\mu Q)^{-1} b, b\right\rangle+\frac{\mu^{2}}{2}\left\langle(I+\mu Q)^{-2} Q b, b\right\rangle+(c-\alpha) \\
& =q\left(-\mu(I+\mu Q)^{-1} b\right)-\alpha
\end{aligned}
$$

Replacing $\mu Q$ by $(I+\mu Q)-I$ in the second term of the right hand side of (3.17) leads to the alternative expressions

$$
\begin{aligned}
\psi_{s r}^{\prime}(\mu) & =-\frac{\mu}{2}\left\langle\left((I+\mu Q)^{-1}+(I+\mu Q)^{-2}\right) b, b\right\rangle+(c-\alpha), \\
& =-\frac{\mu}{2}\left\langle(I+\mu Q)^{-2}(2 I+\mu Q) b, b\right\rangle+(c-\alpha) .
\end{aligned}
$$

Differentiating another time from (3.19) yields

$$
\begin{aligned}
\psi_{s r}^{\prime \prime}(\mu)= & -\frac{1}{2}\left\langle\left((I+\mu Q)^{-1}+(I+\mu Q)^{-2}\right) b, b\right\rangle+ \\
& \frac{\mu}{2}\left\langle\left((I+\mu Q)^{-2}+2(I+\mu Q)^{-3}\right) Q b, b\right\rangle \\
= & -\left\langle(I+\mu Q)^{-3} b, b\right\rangle .
\end{aligned}
$$

Let $\Lambda$ be the set of eigenvalues of $Q, \Lambda^{\star}:=\Lambda \backslash\{0\}$ and, for $\lambda \in \mathbb{R}$, denote by $b_{\lambda}$ the orthogonal projection of $b$ on $\operatorname{ker}(Q-\lambda I)$. From (3.20) we get for all $\mu \in]-1 / \lambda_{\max },+\infty[$

$$
\begin{aligned}
\psi_{s r}^{\prime}(\mu) & =-\frac{\mu}{2} \sum_{\lambda \in \Lambda} \frac{2+\mu \lambda}{(1+\mu \lambda)^{2}}\left\|b_{\lambda}\right\|^{2}+c-\alpha \\
& =-\mu\left\|b_{0}\right\|^{2}+\frac{1}{2} \sum_{\lambda \in \Lambda^{\star}}\left(\frac{1}{\lambda(1+\mu \lambda)^{2}}-\frac{1}{\lambda}\right)\left\|b_{\lambda}\right\|^{2}+c-\alpha .
\end{aligned}
$$

Note that $\psi_{s r}^{\prime}(0)=c-\alpha<0$.

- Easy case: $b \notin \operatorname{ker}\left(Q-\lambda_{\max } I\right)^{\perp}$. Then $b_{\lambda_{\max }} \neq 0$ and, due to (3.23), $\lim _{\mu \rightarrow\left(-1 / \lambda_{\max }\right)^{+}} \psi_{s r}^{\prime}(\mu)=+\infty$. Due to $(3.22)$, it follows that $\psi_{s r}^{\prime}$ is decreasing and $\psi_{s r}$ admits a unique maximizer $\bar{\mu}$ on $]-1 / \lambda_{\max },+\infty[$. It is characterized by $\psi_{s r}^{\prime}(\bar{\mu})=0$ and obviously belongs to $]-1 / \lambda_{\max }, 0[$.

- Hard case: $b \in \operatorname{ker}\left(Q-\lambda_{\max } I\right)^{\perp}$. Then the expressions (3.16)-(3.18) remain true for $\mu=-1 / \lambda_{\max }$, provided that the restriction of $I+\mu Q$ to $\operatorname{ker}(Q-$ $\left.\lambda_{\max } I\right)^{\perp}$ is considered. In particular,

$$
\psi_{s r}^{\prime}\left(-1 / \lambda_{\max }\right)=q\left(\left(\lambda_{\max } I-Q\right)^{\dagger} b\right)
$$

is finite.

If $\psi_{s r}^{\prime}\left(-1 / \lambda_{\max }\right)>0$ (hard case I), then $b \neq 0$. Due to (3.22), it follows that $\psi_{s r}^{\prime}$ is decreasing and $\psi_{s r}$ admits a unique maximizer $\bar{\mu}$ in 
] $-1 / \lambda_{\max },+\infty\left[\right.$, characterized by $\psi_{s r}^{\prime}(\bar{\mu})=0$ and clearly belonging to ]$-1 / \lambda_{\max }, 0[$.

If $\psi_{s r}^{\prime}\left(-1 / \lambda_{\max }\right) \leq 0$ (hard case II), in view of $(3.22)$ and $\psi_{s r}^{\prime}(0)=$ $c-\alpha<0, \psi_{s r}$ is decreasing and $-1 / \lambda_{\max }$ is the unique maximizer of $\psi_{s r}$ in $\left[-1 / \lambda_{\max },+\infty[\right.$.

By Theorem 3.7, the set of solutions of the primal problem is given by

$$
\Xi=\operatorname{argmin} L(., \bar{\mu}) \cap[q=\alpha] .
$$

Here the Lagrangian is

$$
L(\xi, \bar{\mu})=\frac{1}{2}\langle(I+\bar{\mu} Q) \xi, \xi\rangle+\bar{\mu}\langle b, \xi\rangle+\bar{\mu}(c-\alpha) .
$$

In the easy case and the hard case $\mathrm{I}, L(., \bar{\mu})$ is strictly convex since $\bar{\mu}>-1 / \lambda_{\max }$. It admits as unique minimizer

$$
\bar{\xi}=-\bar{\mu}(I+\bar{\mu} Q)^{-1} b .
$$

From (3.18) and $\psi_{s r}^{\prime}(\bar{\mu})=0$ we derive that $q(\bar{\xi})=\frac{1}{2}\langle Q \bar{\xi}, \bar{\xi}\rangle+\langle b, \bar{\xi}\rangle+c=\alpha$.

In the hard case II, we have $\bar{\mu}=-1 / \lambda_{\max }$ and

$$
\operatorname{argmin} L(., \bar{\mu})=\left\{\xi \in \mathcal{H},\left(Q-\lambda_{\text {max }} I\right) \xi=-b\right\} .
$$

Using that $b \in \operatorname{ker}\left(Q-\lambda_{\max } I\right)^{\perp}=\operatorname{im}\left(Q-\lambda_{\max } I\right)$ we obtain

$$
\operatorname{argmin} L(., \bar{\mu})=\left\{\left(\lambda_{\max } I-Q\right)^{\dagger} b\right\}+\operatorname{ker}\left(Q-\lambda_{\max } I\right) .
$$

Observe that $q\left(-\left(Q-\lambda_{\max } I\right)^{\dagger} b\right) \leq \alpha$ confirms that $\Xi \neq \emptyset$.

Remark 3.9. If $f_{\Omega}^{N} \in \operatorname{im} B_{\Omega}$, then the expressions (3.14) and (3.15) can be conveniently rewritten. Indeed, writing $f_{\Omega}^{N}=B_{\Omega} \xi^{N}$ yields $b=Q \xi^{N}$ as well as $c=\frac{1}{2}\left\langle Q \xi^{N}, \xi^{N}\right\rangle$. Plugging these expressions into (3.14) entails after simplification

$$
\psi_{s r}^{\prime}(\mu)=q\left((I+\mu Q)^{-1} \xi^{N}-\xi^{N}\right)-\alpha=C\left(\Omega, B_{\Omega}(I+\mu Q)^{-1} \xi^{N}\right)-\alpha .
$$

To conclude this section, we present an interesting connection between the dual problem (3.13) and a semidefinite programming problem inspired by [10].

We will need the following version of the S-Lemma in Hilbert spaces . It relies on slightly different assumptions from Theorem 3.6, but its statement is contained in the same reference (see again [19] for the original version in Russian).

Theorem 3.10 (S-lemma, [20], Theorem 2.1). Let $s_{i}: \mathcal{H} \rightarrow \mathbb{R}, i=1,2$, be two quadratic functionals of the form $s_{i}: \xi \rightarrow \frac{1}{2}\left\langle S_{i} \xi, \xi\right\rangle+\left\langle d_{i}, \xi\right\rangle+e_{i}, S_{i}: \mathcal{H} \rightarrow \mathcal{H}$ continuous and self-adjoint, $d_{i} \in \mathcal{H}$ and $e_{i} \in \mathbb{R}, i=1,2$. Suppose that there exists $\xi_{+} \in \mathcal{H}$ such that $s_{2}\left(\xi_{+}\right)>0$ and

$$
\forall \xi \in \mathcal{H}, \quad s_{2}(\xi) \geq 0 \Longrightarrow s_{1}(\xi) \geq 0 .
$$

Then there exists $\lambda \geq 0$ such that

$$
\forall \xi \in \mathcal{H}, \quad s_{1}(\xi)-\lambda s_{2}(\xi) \geq 0 .
$$

Moreover, if there exists $\xi_{-}$such that $s_{1}\left(\xi_{-}\right)<0$, one can suppose that $\lambda>0$.

Let us consider the following problem:

$$
\text { Maximize } \rho \text { subject to } \rho \mathbb{B} \subset[q \leq \alpha], \quad \rho \in \mathbb{R}_{+}^{*},
$$


whose (unique) solution is $\rho=\operatorname{dist}(0,[q=\alpha])=\operatorname{dist}(0,[q>\alpha]) \in \mathbb{R}_{+}^{\star}$ since $c=q(0)<\alpha$ and $Q \geq 0, Q \neq 0$ so there exists $\xi_{0}$ such that $q\left(\xi_{0}\right) \geq \alpha$. Yet we have for all $\rho>0$

$$
\begin{aligned}
\rho \mathbb{B} \subset[q \leq \alpha] & \Longleftrightarrow \forall \xi \in \mathcal{H},\|\xi\| \leq \rho \Rightarrow q(\xi) \leq \alpha, \\
& \Longleftrightarrow \forall \xi \in \mathcal{H},\|\xi\| \leq 1 \Rightarrow q(\rho \xi) \leq \alpha, \\
& \Longleftrightarrow \forall \xi \in \mathcal{H}, 1-\|\xi\|^{2} \geq 0 \Rightarrow \alpha-q(\rho \xi) \geq 0 .
\end{aligned}
$$

As $1-\|\xi\|^{2}=1>0$ for $\xi=0$ and $\alpha-q(\rho \xi)<0$ for some $\xi$, using Theorem 3.10, one obtains

$$
\rho \mathbb{B} \subset[q \leq \alpha] \Longleftrightarrow \exists \lambda>0, \forall \xi \in \mathcal{H}, \alpha-q(\rho \xi)-\lambda\left[1-\|\xi\|^{2}\right] \geq 0 .
$$

But $q(\xi)$ can be expressed as

$$
q(\xi)=\max _{v \in \mathcal{V}_{\Omega}}-\frac{1}{2}\left\langle A_{\Omega} v, v\right\rangle+\left\langle f_{\Omega}^{N}+B_{\Omega} \xi, v\right\rangle,
$$

whereby

$$
\begin{aligned}
\alpha-q(\rho \xi)-\lambda\left[1-\|\xi\|^{2}\right] & \geq 0 \Longleftrightarrow \\
\forall v & \in \mathcal{V}_{\Omega}, \frac{1}{2}\left\langle A_{\Omega} v, v\right\rangle+\lambda\|\xi\|^{2}-\rho\left\langle B_{\Omega} \xi, v\right\rangle-\left\langle f_{\Omega}^{N}, v\right\rangle+\alpha-\lambda \geq 0 .
\end{aligned}
$$

Changing $(\xi, v)$ into $\left(s^{-1} \xi, s^{-1} v\right)$ for any $s \neq 0$, one also has

$$
\begin{aligned}
\forall \xi \in \mathcal{H}, \alpha-q(\rho \xi)-\lambda\left[1-\|\xi\|^{2}\right] \geq 0 & \Longleftrightarrow \forall(v, \xi, s) \in \mathcal{V}_{\Omega} \times \mathcal{H} \times \mathbb{R}, \\
& \frac{1}{2}\left\langle A_{\Omega} v, v\right\rangle+\lambda\|\xi\|^{2}-\rho\left\langle B_{\Omega} \xi, v\right\rangle \\
-s\left\langle f_{\Omega}^{N}, v\right\rangle+(\alpha-\lambda) s^{2} \geq 0, & \\
& \Longleftrightarrow\left[\begin{array}{ccc}
A_{\Omega} & \rho B_{\Omega} & \left(f_{\Omega}^{N}\right)^{*} \\
\rho B_{\Omega}^{*} & 2 \lambda I & 0 \\
f_{\Omega}^{N} & 0 & 2(\alpha-\lambda)
\end{array}\right] \geq 0,
\end{aligned}
$$

where $\left(f_{\Omega}^{N}\right)^{*}: \mathbb{R} \rightarrow \mathcal{V}_{\Omega}^{\prime}$ is defined as $s \rightarrow s f_{\Omega}^{N}$. Finaly

$$
\rho \mathbb{B} \subset[q \leq \alpha] \Longleftrightarrow \exists \lambda>0,\left[\begin{array}{ccc}
A_{\Omega} & \rho B_{\Omega} & \left(f_{\Omega}^{N}\right)^{*} \\
\rho B_{\Omega}^{*} & 2 \lambda I & 0 \\
f_{\Omega}^{N} & 0 & 2(\alpha-\lambda)
\end{array}\right] \geq 0 .
$$

Therefore, $\bar{\rho}>0$ is the solution of (3.24) if and only if there exists $\bar{\lambda}>0$ such that $(\bar{\rho}, \bar{\lambda})$ is solution of:

$$
\text { Maximize } \rho \text { subject to }\left[\begin{array}{ccc}
A_{\Omega} & \rho B_{\Omega} & \left(f_{\Omega}^{N}\right)^{*} \\
\rho B_{\Omega}^{*} & \lambda I & 0 \\
f_{\Omega}^{N} & 0 & 2 \alpha-\lambda
\end{array}\right] \geq 0, \quad \rho, \lambda \in \mathbb{R}_{+}^{\star} .
$$

Note that the above matrix is affine in $(\rho, \lambda)$ and that, as Problem (3.24) admits a solution, it is also the case for Problem (3.26).

Proposition 3.11. Problem (3.26) admits a unique solution $(\bar{\rho}, \bar{\lambda})$. This solution is such that $\bar{\rho}=\operatorname{dist}(0,[q>\alpha])$ and $-\bar{\rho}^{2} / \bar{\lambda}$ is the solution of the dual problem (3.13). 
Proof. Let $(\bar{\rho}, \bar{\lambda})$ be a solution of (3.26). The constraint implies

$$
\forall \xi \in \mathcal{H}, \quad \alpha-q(\bar{\rho} \xi)-\frac{\bar{\lambda}}{2}\left[1-\|\xi\|^{2}\right] \geq 0 .
$$

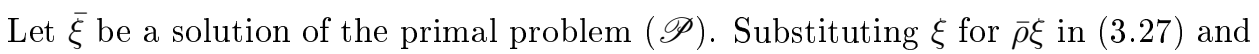
multiplying by $\bar{\rho}^{2} / \bar{\lambda}$ we arrive at

$$
\forall \xi \in \mathcal{H}, \quad \frac{1}{2}\|\xi\|^{2}-\frac{\bar{\rho}^{2}}{\bar{\lambda}}[q(\xi)-\alpha] \geq \frac{1}{2} \bar{\rho}^{2}=\frac{1}{2}\|\bar{\xi}\|^{2} .
$$

As $q(\bar{\xi})=\alpha$ we can write

$$
\forall \xi \in \mathcal{H}, \quad \frac{1}{2}\|\xi\|^{2}-\frac{\bar{\rho}^{2}}{\bar{\lambda}}[q(\xi)-\alpha] \geq \frac{1}{2}\left\|\bar{\xi}^{2}\right\|-\frac{\bar{\rho}^{2}}{\bar{\lambda}}[q(\bar{\xi})-\alpha] .
$$

It follows that

$$
\bar{\xi} \in \operatorname{argmin} L\left(.,-\bar{\rho}^{2} / \bar{\lambda}\right) \cap\left[L\left(.,-\bar{\rho}^{2} / \bar{\lambda}\right)=\frac{1}{2}\|\cdot\|^{2}\right]
$$

thus, by virtue of (A.7), $-\bar{\rho}^{2} / 2 \bar{\lambda}$ is the solution of the dual problem (3.13). The unicity of the solution of the dual problem and the implication just proved imply the unicity of the solution of Problem (3.26).

3.4. Expression of the critical loads for the robust compliance in the worst case sense. Here, using again the notation (2.1), the primal problem $(\mathscr{P})$ and the dual problem $(\mathscr{D})$ are equivalent, respectively, to:

$$
\text { Minimize }-q(\xi)=-\frac{1}{2}\langle Q \xi, \xi\rangle-\langle b, \xi\rangle-c \quad \text { subject to }\|\xi\|=r, \quad \xi \in \mathcal{H},
$$

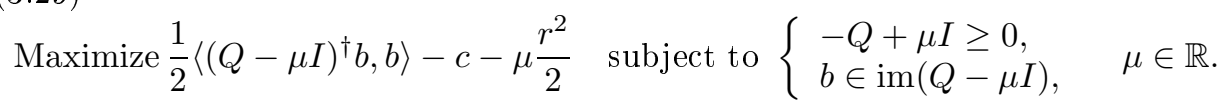

The largest eigenvalue of $Q$ is still denoted by $\lambda_{\max }$. Applying Theorem 3.7 similarly to Theorem 3.8 provides the following result.

Theorem 3.12. The primal problem (3.28) and the dual problem (3.29) have the same optimal values. The dual problem (3.29) admits a unique solution $\bar{\mu} \in \mathbb{R}$, which can be computed in the following way.

- Easy case: $b \notin \operatorname{ker}\left(Q-\lambda_{\max } I\right)^{\perp}$. Then $\bar{\mu}$ is the unique solution in $] \lambda_{\max },+\infty[$ of the equation

$$
\left\|(-Q+\mu I)^{-1} b\right\|=r .
$$

- Hard case $I: b \in \operatorname{ker}\left(Q-\lambda_{\max } I\right)^{\perp}$ and $\left\|\left(-Q+\lambda_{\max } I\right)^{\dagger} b\right\|>r$. Then $\bar{\mu}$ is also the unique solution in $] \lambda_{\max },+\infty[$ of (3.30).

- Hard case II: $b \in \operatorname{ker}\left(Q-\lambda_{\max } I\right)^{\perp}$ and $\left\|\left(-Q+\lambda_{\max } I\right)^{\dagger} b\right\| \leq r$. Then $\bar{\mu}=\lambda_{\max }$.

The set $\Xi$ of solutions of the primal problem (3.28) is given by the following expressions.

- Easy case and Hard case I. There is a unique critical load given by

$$
\Xi=\left\{(-Q+\bar{\mu} I)^{-1} b\right\} .
$$


- Hard case II. The set of critical loads is

$$
\Xi=\left[\left\{\left(-Q+\lambda_{\max } I\right)^{\dagger} b\right\}+\operatorname{ker}\left(Q-\lambda_{\max } I\right)\right] \cap[\|.\|=r] .
$$

As in the preceding subsection, we present a connection between the dual problem (3.29) and a semidefinite programming problem. Let us consider the following problem:

$$
\text { Minimize } \alpha \text { subject to } r \mathbb{B} \subset[q \leq \alpha], \quad \alpha \in \mathbb{R},
$$

where $r>0$ and whose (unique) solution is $\alpha=\sup _{\|\xi\| \leq r} q(\xi)$.

Therefore, using $(3.25), \bar{\alpha}$ is the solution of (3.31) if and only if there exists $\bar{\lambda}>0$ such that $(\bar{\alpha}, \bar{\lambda})$ is solution of:

$$
\text { Minimize } \alpha \text { subject to }\left[\begin{array}{ccc}
A_{\Omega} & r B_{\Omega} & \left(f_{\Omega}^{N}\right)^{*} \\
r B_{\Omega}^{*} & \lambda I & 0 \\
f_{\Omega}^{N} & 0 & 2 \alpha-\lambda
\end{array}\right] \geq 0, \quad \alpha \in \mathbb{R}, \lambda \in \mathbb{R}_{+}^{*} .
$$

Note that the above matrix is affine in $(\alpha, \lambda)$ and that, as Problem (3.31) admits a solution, the same holds for Problem (3.32). Similarly to Proposition 3.11 we obtain the following.

Proposition 3.13. Problem (3.32) admits a unique solution $(\bar{\alpha}, \bar{\lambda})$. This solution is such that $\bar{\alpha}=\sup _{\|\xi\| \leq r} q(\xi)$ and $\bar{\lambda} / r^{2}$ is the solution of the dual problem (3.29).

\section{HADAMARD SEMIDERIVATIVE OF THE ROBUST CRITERIA}

The functionals $j_{s r}(\Omega)$ and $j_{w c}(\Omega)$ can be written as

$$
j_{s r}(\Omega)=J_{s r}\left(w_{\Omega}\right), \quad j_{w c}(\Omega)=J_{w c}\left(w_{\Omega}\right),
$$

with $w_{\Omega}=\left(Q_{\Omega}, b_{\Omega}, c_{\Omega}\right)$ defined by $(2.1), w_{\Omega} \in W:=\mathcal{K}_{s}(\mathcal{H}) \times \mathcal{H} \times \mathbb{R}$, where $\mathcal{K}_{s}(\mathcal{H})$ stands for the space of self-adjoint compact linear operators from $\mathcal{H}$ into itself. Moreover $J_{s r}, J_{w c}: W \rightarrow \mathbb{R} \cup\{+\infty\}$ are defined, for $w \in W$, by

$$
J_{s r}(w)=\inf _{q(w, \xi)=\alpha} \frac{1}{2}\|\xi\|^{2},
$$

and

$$
J_{w c}(w)=\sup _{\|\xi\|=r} q(w, \xi)
$$

with, for $w=(Q, b, c) \in W$,

$$
q(w, \xi)=\frac{1}{2}\langle Q \xi, \xi\rangle+\langle b, \xi\rangle+c
$$

In view of (4.1), calculating the shape derivative or the topological derivative of $j_{s r}$ or $j_{w c}$ requires to calculate the Hadamard semiderivative of $J_{s r}$ or $J_{w c}$. This is the aim of this section. The sensitivity of $w$ with respect to $\Omega$, specifically its topological derivative, will be studied in Section 5 .

Our approach, on one hand, adapts the proof of Theorem 4.24 in [12] to a slightly different context, while, on the other hand, it shows that the modified assumptions of this Theorem, in particular the strong assumption (iii), are fulfilled.

In all this section, we consider some $\bar{w}=(\bar{Q}, \bar{b}, \bar{c}) \in W$ with $\bar{Q}: \mathcal{H} \rightarrow \mathcal{H}$ a selfajoint compact operator such that $\bar{Q} \geq 0, \bar{Q} \neq 0$ and some $\alpha, r \in \mathbb{R}$ with $\bar{c}<\alpha$, $r>0$. 
4.1. Hadamard semiderivatives. Let $X$ be a Banach space and consider a fonction $f: X \rightarrow \mathbb{R} \cup\{-\infty,+\infty\}$. If $z, d \in X$ and $f(z) \in \mathbb{R}$ the upper and lower Hadamard semiderivatives of $f$ at point $z$ in direction $d$ are defined, respectively, as

$$
f_{+}^{\prime}(z, d)=\limsup _{\substack{t \downarrow 0 \\ v \rightarrow d}} \frac{f(z+t v)-f(z)}{t}, \quad f_{-}^{\prime}(z, d)=\liminf _{\substack{t \downarrow 0 \\ v \rightarrow d}} \frac{f(z+t v)-f(z)}{t} .
$$

If $f_{+}^{\prime}(z, d)=f_{-}^{\prime}(z, d)$, their common value is called the Hadamard semiderivative of $f$ at point $z$ in direction $d$, denoted by $f^{\prime}(z, d)$.

4.2. Hadamard semiderivative of the compliance robustness. Let $w \in W$ and denote by $\Xi(w)$ the minimizing set of (4.2). Recall that the Lagrangian of this problem is

$$
L(w, \xi, \mu)=\frac{1}{2}\|\xi\|^{2}+\mu(q(w, \xi)-\alpha)
$$

and that the dual criterion is

$$
g(w, \mu)=\inf _{\xi \in \mathcal{H}} L(w, \xi, \mu)
$$

We denote by $\bar{\mu}$ the unique solution of the dual problem for $\bar{w}$, i.e. the unique element of $\operatorname{argmax} g(\bar{w},$.$) .$

Lemma 4.1. Let $\bar{\xi} \in \mathcal{H}$ be such that $q(\bar{w}, \bar{\xi})=\alpha$. There exists a neighborhood $\mathcal{W}$ of $\bar{w}$ and a function $S_{\bar{w}, \bar{\xi}}: \mathcal{W} \rightarrow \mathcal{H}$ of class $\mathscr{C}^{\infty}$ such that $S_{\bar{w}, \bar{\xi}}(\bar{w})=\bar{\xi}$ and

$$
q\left(w, S_{\bar{w}, \bar{\xi}}(w)\right)=\alpha \quad \forall w \in \mathcal{W} .
$$

Proof. Consider the function $F:(w, s) \in W \times \mathbb{R} \mapsto q(w, s \bar{\xi})-\alpha$, which is clearly of class $\mathscr{C}^{\infty}$. We have

$$
\partial_{s} F(\bar{w}, 1)=\langle\bar{Q} \bar{\xi}, \bar{\xi}\rangle+\langle\bar{b}, \bar{\xi}\rangle=\frac{1}{2}\langle\bar{Q} \bar{\xi}, \bar{\xi}\rangle-(\bar{c}-\alpha),
$$

due to $q(\bar{w}, \bar{\xi})=\alpha$. Using that $\bar{Q} \geq 0$ and $\bar{c}<\alpha$ we infer $\partial_{s} F(\bar{w}, 1)>0$. The implicit function theorem leads to the result.

Lemma 4.2. We have, for any $\bar{h} \in W$,

$$
\left(J_{s r}\right)_{+}^{\prime}(\bar{w}, \bar{h}) \leq \inf _{\bar{\xi} \in \Xi(\bar{w})} \partial_{w} L(\bar{w}, \bar{\xi}, \bar{\mu}) \bar{h} .
$$

Proof. Choose an arbitrary $\bar{\xi} \in \Xi(\bar{w})$. Let $\left(t_{n}, h_{n}\right) \in \mathbb{R}_{+}^{*} \times W$ be such that $t_{n} \rightarrow 0$, $h_{n} \rightarrow \bar{h}$, and

$$
\left(J_{s r}\right)_{+}^{\prime}(\bar{w}, \bar{h})=\lim _{n \rightarrow+\infty} \frac{J_{s r}\left(\bar{w}+t_{n} h_{n}\right)-J_{s r}(\bar{w})}{t_{n}} .
$$

We assume that $n$ is large enough so that $w_{n}:=\bar{w}+t_{n} h_{n} \in \mathcal{W}$. Denoting $\xi_{n}:=$ $S_{\bar{w}, \bar{\xi}}\left(w_{n}\right)$, we have $q\left(w_{n}, \xi_{n}\right)=\alpha$, hence (4.2) entails

$$
J_{s r}\left(w_{n}\right) \leq \frac{1}{2}\left\|\xi_{n}\right\|^{2}=L\left(w_{n}, \xi_{n}, \mu\right)
$$

for any $\mu \in \mathbb{R}$. As $\bar{\xi} \in \Xi(\bar{w})$, (4.2) also yields

$$
J_{s r}(\bar{w})=\frac{1}{2}\|\bar{\xi}\|^{2}=L(\bar{w}, \bar{\xi}, \mu) .
$$

Therefore we have

$$
\frac{J_{s r}\left(w_{n}\right)-J_{s r}(\bar{w})}{t_{n}} \leq \frac{L\left(w_{n}, \xi_{n}, \mu\right)-L(\bar{w}, \bar{\xi}, \mu)}{t_{n}} .
$$


For all $w$ in a neighborhood of $\bar{w}$ we set

$$
\Phi(w)=L\left(w, S_{\bar{w}, \bar{\xi}}(w), \mu\right) .
$$

We have

$$
L\left(w_{n}, \xi_{n}, \mu\right)-L(\bar{w}, \bar{\xi}, \mu)=\Phi\left(w_{n}\right)-\Phi(\bar{w})=d \Phi(\bar{w})\left(t_{n} h_{n}\right)+o_{n \rightarrow+\infty}\left(t_{n} h_{n}\right),
$$

since $\Phi$ is Fréchet differentiable by composition. The chain rule gives, for $v \in \mathcal{H}$,

$$
d \Phi(\bar{w})(v)=\partial_{w} L(\bar{w}, \bar{\xi}, \mu) v+\partial_{\xi} L(\bar{w}, \bar{\xi}, \mu)\left(d S_{\bar{w}, \bar{\xi}}(\bar{w}) v\right) .
$$

We can choose $\mu=\bar{\mu}$, for which it holds $\partial_{\xi} L(\bar{w}, \bar{\xi}, \bar{\mu})=0$. We arrive at $d \Phi(\bar{w})(v)=$ $\partial_{w} L(\bar{w}, \bar{\xi}, \bar{\mu}) v$, and

$$
\frac{J_{s r}\left(\bar{w}+t_{n} h_{n}\right)-J_{s r}(\bar{w})}{t_{n}} \leq \partial_{w} L(\bar{w}, \bar{\xi}, \bar{\mu}) h_{n}+o(1) .
$$

Passing to the limit yields

$$
\left(J_{s r}\right)_{+}^{\prime}(\bar{w}, \bar{h}) \leq \partial_{w} L(\bar{w}, \bar{\xi}, \bar{\mu}) \bar{h} .
$$

This being true for any $\bar{\xi} \in \Xi(\bar{w})$, we arrive at the desired result.

Lemma 4.3. For any $\bar{h} \in W$ we have

$$
\left(J_{s r}\right)_{-}^{\prime}(\bar{w}, \bar{h}) \geq \inf _{\bar{\xi} \in \Xi(\bar{w})} \partial_{w} L(\bar{w}, \bar{\xi}, \bar{\mu}) \bar{h} .
$$

Proof. Let $\left(t_{n}, h_{n}\right) \in \mathbb{R}_{+}^{*} \times W$ be such that $t_{n} \rightarrow 0, h_{n} \rightarrow \bar{h}$, and

$$
\left(J_{s r}\right)_{-}^{\prime}(\bar{w}, \bar{h})=\lim _{n \rightarrow+\infty} \frac{J_{s r}\left(\bar{w}+t_{n} h_{n}\right)-J_{s r}(\bar{w})}{t_{n}} .
$$

For all $n$ we set $w_{n}=\bar{w}+t_{n} h_{n}$ and choose some $\xi_{n} \in \Xi\left(w_{n}\right)$ (cf. Remark 3.2).

Step 1. We choose an arbitrary $\xi \in \mathcal{H}$ such that $q(\bar{w}, \xi)=\alpha$. By Lemma 4.1, there exists a neighborhood $\mathcal{W}$ of $\bar{w}$ and a function $S_{\bar{w}, \xi}: \mathcal{W} \rightarrow \mathcal{H}$ of class $\mathscr{C}^{\infty}$ such that $S_{\bar{w}, \xi}(\bar{w})=\xi$ and

$$
q\left(w, S_{\bar{w}, \xi}(w)\right)=\alpha \quad \forall w \in \mathcal{W} .
$$

Since $w_{n} \rightarrow \bar{w}$, it holds for $n$ large enough $q\left(w_{n}, S_{\bar{w}, \xi}\left(w_{n}\right)\right)=\alpha$, hence

$$
\frac{1}{2}\left\|\xi_{n}\right\|^{2} \leq \frac{1}{2}\left\|S_{\bar{w}, \xi}\left(w_{n}\right)\right\|^{2} .
$$

This shows that the sequence $\left(\xi_{n}\right)$ is bounded. Therefore there exists $\bar{\xi} \in \mathcal{H}$ such that $\xi_{n} \rightarrow \bar{\xi}$ weakly for some non-relabeled subsequence.

Step 2. We shall show that $\bar{\xi} \in \Xi(\bar{w})$. From $w_{n}=\bar{w}+t_{n} h_{n}$ and $q\left(w_{n}, \xi_{n}\right)=\alpha$, denoting $h_{n}=\left(Q_{n}, b_{n}, c_{n}\right)$, we obtain

$$
q\left(w_{n}, \xi_{n}\right)=\frac{1}{2}\left\langle\left(\bar{Q}+t_{n} Q_{n}\right) \xi_{n}, \xi_{n}\right\rangle+\left\langle\bar{b}+t_{n} b_{n}, \xi_{n}\right\rangle+\bar{c}+t_{n} c_{n}=\alpha .
$$

We have $\bar{Q}+t_{n} Q_{n} \rightarrow \bar{Q}, \bar{Q}$ compact, $\bar{b}+t_{n} b_{n} \rightarrow \bar{b}, \bar{c}+t_{n} c_{n} \rightarrow \bar{c}$ and $\xi_{n} \rightarrow \bar{\xi}$. Hence $\left(\bar{Q}+t_{n} Q_{n}\right) \xi_{n} \rightarrow \bar{Q} \bar{\xi}$ strongly and $\frac{1}{2}\left\langle\left(\bar{Q}+t_{n} Q_{n}\right) \xi_{n}, \xi_{n}\right\rangle \rightarrow \frac{1}{2}\langle\bar{Q} \bar{\xi}, \bar{\xi}\rangle$. Passing to the limit in (4.5) yields

$$
\frac{1}{2}\langle\bar{Q} \bar{\xi}, \bar{\xi}\rangle+\langle\bar{b}, \bar{\xi}\rangle+\bar{c}=q(\bar{w}, \bar{\xi})=\alpha .
$$

Moreover, since $\xi_{n} \rightarrow \bar{\xi}$ and $S_{\bar{w}, \xi}\left(w_{n}\right) \rightarrow S_{\bar{w}, \xi}(\bar{w})=\xi$, (4.4) entails

$$
\frac{1}{2}\|\bar{\xi}\|^{2} \leq \frac{1}{2} \liminf _{n \rightarrow+\infty}\left\|\xi_{n}\right\|^{2} \leq \frac{1}{2} \liminf _{n \rightarrow+\infty}\left\|S_{\bar{w}, \xi}\left(w_{n}\right)\right\|^{2}=\frac{1}{2}\|\xi\|^{2} .
$$


This proves that $\bar{\xi} \in \Xi(\bar{w})$.

Step 3. From Theorem 3.7, we have $J_{s r}(\bar{w})=g(\bar{w}, \bar{\mu})=\inf _{\xi \in \mathcal{H}} L(\bar{w}, \xi, \bar{\mu})$. It follows that $J_{s r}(\bar{w}) \leq L\left(\bar{w}, \xi_{n}, \bar{\mu}\right)$. As $\xi_{n} \in \Xi\left(\bar{w}_{n}\right)$, we have $J_{s r}\left(w_{n}\right)=\frac{1}{2}\left\|\xi_{n}\right\|^{2}=$ $L\left(w_{n}, \xi_{n}, \bar{\mu}\right)$. We arrive at

$$
\frac{J_{s r}\left(w_{n}\right)-J_{s r}(\bar{w})}{t_{n}} \geq \frac{L\left(w_{n}, \xi_{n}, \bar{\mu}\right)-L\left(\bar{w}, \xi_{n}, \bar{\mu}\right)}{t_{n}} .
$$

Yet we have

$$
\frac{L\left(w_{n}, \xi_{n}, \bar{\mu}\right)-L\left(\bar{w}, \xi_{n}, \bar{\mu}\right)}{t_{n}}=\bar{\mu}\left[\frac{1}{2}\left\langle Q_{n} \xi_{n}, \xi_{n}\right\rangle+\left\langle b_{n}, \xi_{n}\right\rangle+c_{n}\right] .
$$

Denoting $\bar{h}=(Q, b, c)$ and using that $Q_{n} \rightarrow Q$ compact, $b_{n} \rightarrow b, c_{n} \rightarrow c$ and $\xi_{n} \rightarrow \bar{\xi}$ weakly, one obtains

$$
\frac{L\left(w_{n}, \xi_{n}, \bar{\mu}\right)-L\left(\bar{w}, \xi_{n}, \bar{\mu}\right)}{t_{n}} \longrightarrow \bar{\mu}\left[\frac{1}{2}\langle Q \bar{\xi}, \bar{\xi}\rangle+\langle b, \xi\rangle+c\right]=\partial_{w} L(\bar{w}, \bar{\xi}, \bar{\mu}) \bar{h} .
$$

It follows that

$$
\left(J_{s r}\right)_{-}^{\prime}(\bar{w}, \bar{h})=\lim _{n \rightarrow+\infty} \frac{J_{s r}\left(\bar{w}+t_{n} h_{n}\right)-J_{s r}(\bar{w})}{t_{n}} \geq \partial_{w} L(\bar{w}, \bar{\xi}, \bar{\mu}) \bar{h} .
$$

The proof is therefore complete.

From Lemmas 4.2 and 4.3, we derive the following.

Theorem 4.4. For any $\bar{h} \in W, J_{\text {sr }}$ admits a Hadamard semiderivative at point $\bar{w}$ in the direction $\bar{h}$ given by

$$
J_{s r}^{\prime}(\bar{w}, \bar{h})=\inf _{\bar{\xi} \in \Xi(\bar{w})} \partial_{w} L(\bar{w}, \bar{\xi}, \bar{\mu}) \bar{h}=\inf _{\bar{\xi} \in \Xi(\bar{w})} \bar{\mu} q(\bar{h}, \bar{\xi}),
$$

where $\bar{\mu}$ is the unique solution of the dual problem (3.13).

4.3. Hadamard semiderivative of the robust compliance in the worst case sense. We denote by $\Xi(\bar{w})$ the maximizing set of (4.3) with $w=\bar{w}$, which has been obtained in Theorem 3.12. Slightly adapting the proof of Theorem 4.13 in [12], one obtains the following result.

Theorem 4.5. For any $\bar{h} \in W$, the worst case functional $J_{w c}$ admits a Hadamard semiderivative at point $\bar{w}$ in the direction $\bar{h}$ given by

$$
J_{w c}^{\prime}(\bar{w}, \bar{h})=\sup _{\bar{\xi} \in \Xi(\bar{w})} \partial_{w} q(\bar{w}, \bar{\xi}) \bar{h}=\sup _{\bar{\xi} \in \Xi(\bar{w})} q(\bar{h}, \bar{\xi}) .
$$

\section{Topological Derivative of the Robust CRITERia}

Consider a reference domain $\Omega=\Omega_{0} \in \mathcal{E}$ and a family of perturbed domains $\left(\Omega_{t}\right)_{t>0}$ such that, for all $t$ small enough, $\Omega_{t} \in \mathcal{E}$. We choose a nominal load of the form $f_{\Omega}^{N}=B_{\Omega} \xi^{N} \in \mathcal{V}_{\Omega}^{\prime}$ with $\xi^{N} \in \mathcal{H}$. As in the previous sections, set $w_{\Omega}=\left(Q_{\Omega}, b_{\Omega}, c_{\Omega}\right)$ with

$$
\begin{aligned}
Q_{\Omega}=B_{\Omega}^{*} A_{\Omega}^{-1} B_{\Omega}, \quad b_{\Omega}=B_{\Omega}^{*} A_{\Omega}^{-1} B_{\Omega} \xi^{N} & =Q_{\Omega} \xi^{N}, \\
c_{\Omega} & =\frac{1}{2}\left\langle B_{\Omega}^{*} A_{\Omega}^{-1} B_{\Omega} \xi^{N}, \xi^{N}\right\rangle=\frac{1}{2}\left\langle Q_{\Omega} \xi^{N}, \xi^{N}\right\rangle .
\end{aligned}
$$

We make the following assumption, which will be verified for specific problems in Section 6. 
Assumption 5.1. There exists $\delta>0$ and a self-adjoint linear operator $G_{\Omega}: \mathcal{H} \rightarrow$ $\mathcal{H}$ such that

$$
\left\langle Q_{\Omega_{t}} \xi, \xi\right\rangle-\left\langle Q_{\Omega} \xi, \xi\right\rangle=t\left\langle G_{\Omega} \xi, \xi\right\rangle+O\left(t^{1+\delta}\right) \quad \forall \xi \in \mathcal{H} .
$$

Lemma 5.2. The function $t \in \mathbb{R}_{+} \mapsto Q_{\Omega_{t}} \in \mathcal{L}(\mathcal{H})$ admits a right derivative at 0 given by

$$
\frac{d}{d t}\left[Q_{\Omega_{t}}\right]_{\mid t=0}=G_{\Omega}
$$

Proof. Assumption 5.1 and the polarization identity entail

$$
\left\langle Q_{\Omega_{t}} \xi, \zeta\right\rangle-\left\langle Q_{\Omega}, \xi, \zeta\right\rangle=t\left\langle G_{\Omega} \xi, \zeta\right\rangle+O\left(t^{1+\delta}\right) \quad \forall \xi, \zeta \in \mathcal{H},
$$

that is,

$$
\forall \xi, \zeta \in \mathcal{H}, \quad \sup _{t>0} t^{-\delta}\left|\left\langle\left(\frac{Q_{\Omega_{t}}-Q_{\Omega}}{t}-G_{\Omega}\right) \xi, \zeta\right\rangle\right|<+\infty .
$$

By the Banach-Steinhaus theorem we obtain

$$
\forall \xi \in \mathcal{H}, \quad \sup _{t>0} t^{-\delta}\left\|\left(\frac{Q_{\Omega_{t}}-Q_{\Omega}}{t}-G_{\Omega}\right) \xi\right\|_{\mathcal{H}}<+\infty .
$$

Another application of the Banach-Steinhaus theorem yields

$$
\sup _{t>0} t^{-\delta}\left\|\frac{Q_{\Omega_{t}}-Q_{\Omega}}{t}-G_{\Omega}\right\|_{\mathcal{L}(\mathcal{H})}<+\infty
$$

In particular we have

$$
\lim _{t \downarrow 0}\left\|\frac{Q_{\Omega_{t}}-Q_{\Omega}}{t}-G_{\Omega}\right\|_{\mathcal{L}(\mathcal{H})}=0,
$$

and the proof is achieved.

The following theorem states the right derivative of $t \rightarrow j_{s r}\left(\Omega_{t}\right)$.

Theorem 5.3. The function $t \mapsto j_{\text {sr }}\left(\Omega_{t}\right)$ admits a right derivative at 0 given by

$$
\frac{d}{d t}\left[j_{s r}\left(\Omega_{t}\right)\right]_{\mid t=0}=\inf _{\bar{\xi} \in \Xi} \frac{1}{2} \bar{\mu}\left\langle\xi^{N}+\bar{\xi}, G_{\Omega}\left(\xi^{N}+\bar{\xi}\right)\right\rangle,
$$

where $\Xi$ is the set of solutions of the primal problem (3.12) and $\bar{\mu}$ is the solution of the dual problem (3.13).

Proof. By Lemma 5.2, we get that the map $t \mapsto w_{\Omega_{t}}$ admits a right derivative at 0 given by

$$
\frac{d}{d t}\left[Q_{\Omega_{t}}\right]_{\mid t=0}=G_{\Omega}, \quad \frac{d}{d t}\left[b_{\Omega_{t}}\right]_{\mid t=0}=G_{\Omega} \xi^{N}, \quad \frac{d}{d t}\left[c_{\Omega_{t}}\right]_{\mid t=0}=\frac{1}{2}\left\langle G_{\Omega} \xi^{N}, \xi^{N}\right\rangle .
$$

Next, with the notation of Section 4 , we have

$$
j_{s r}\left(\Omega_{t}\right)=J_{s r}\left(w_{\Omega_{t}}\right) .
$$

By composition (see, e.g., [12] Proposition 2.47), the function $t \mapsto j_{s r}\left(\Omega_{t}\right)$ admits a right derivative at 0 given by

$$
\frac{d}{d t}\left[j_{s r}\left(\Omega_{t}\right)\right]_{\mid t=0}=J_{s r}^{\prime}\left(w_{\Omega}, \frac{d}{d t}\left[w_{\Omega_{t}}\right]_{\mid t=0}\right) .
$$


Theorem 4.4 yields

$$
\frac{d}{d t}\left[j_{s r}\left(\Omega_{t}\right)\right]_{\mid t=0}=\inf _{\bar{\xi} \in \Xi} \bar{\mu}\left[\frac{1}{2}\left\langle\frac{d}{d t}\left[Q_{\Omega_{t}}\right]_{\mid t=0} \bar{\xi}, \bar{\xi}\right\rangle+\left\langle\frac{d}{d t}\left[b_{\Omega_{t}}\right]_{\mid t=0}, \bar{\xi}\right\rangle+\frac{d}{d t}\left[c_{\Omega_{t}}\right]_{\mid t=0}\right] .
$$

Using (5.2) we arrive at

$$
\frac{d}{d t}\left[j_{s r}\left(\Omega_{t}\right)\right]_{\mid t=0}=\inf _{\bar{\xi} \in \Xi} \bar{\mu}\left[\frac{1}{2}\left\langle G_{\Omega} \bar{\xi}, \bar{\xi}\right\rangle+\left\langle G_{\Omega} \xi^{N}, \bar{\xi}\right\rangle+\frac{1}{2}\left\langle G_{\Omega} \xi^{N}, \xi^{N}\right\rangle\right] .
$$

A rearrangement completes the proof.

\section{Algorithm}

6.1. Problem setting. In the examples we will present we want to minimize

$$
\mathcal{J}(\Omega):=\Phi\left(j_{s r}(\Omega)\right)+\ell|\Omega|,
$$

where $\Phi: \mathbb{R}_{+}^{*} \rightarrow \mathbb{R}$ is a smooth and decreasing function, $\ell$ is a user-given Lagrange multiplier, and $|\Omega|$ is the Lebesgue measure of $\Omega$. In order to maintain $j_{s r}(\Omega)$ positive during the iterations, we further assume that $\lim _{t \rightarrow 0} \Phi(t)=+\infty$. In our computations, for numerical purposes, we have used the function with moderate growth $\Phi(t)=-\log t$.

We choose $\mathcal{E}$ as the set of all subdomains of a fixed "hold-all" domain $D \subset$ $\mathbb{R}^{N}$. Our model problem is that of linear elasticity, with the following standard framework. The domain $\Omega$ is occupied by an elastic material of unitary Young modulus, and its complement $D \backslash \Omega$ is filled with a weak phase, i.e., a fictitious material with small Young modulus $\varepsilon$. This permits to formulate the equilibrium equations, represented by the operator $A_{\Omega}$, in the fixed domain $D$. Therefore the function space $\mathcal{V}_{\Omega}$ is the subspace $H_{D}^{1}(D)^{N}$ including the Dirichlet boundary condition on the appropriate part $\Gamma_{D}$ of $\partial \Omega$.

For some $\hat{x} \in D \backslash \partial \Omega$, we consider the topological perturbation

$$
\Omega_{t}= \begin{cases}\Omega \backslash \overline{\mathbb{B}(\hat{x}, \rho(t))} & \text { if } \quad \hat{x} \in \Omega, \\ (\Omega \cup \mathbb{B}(\hat{x}, \rho(t))) \cap D & \text { if } \quad \hat{x} \in D \backslash \bar{\Omega},\end{cases}
$$

with $\rho(t)=t^{1 / N}$.

6.2. Optimality condition. The derivative of $\mathcal{J}\left(\Omega_{t}\right)$ with respect to $t$ is the so called topological derivative. It is given by the chain rule:

$$
g_{\Omega}(\hat{x}):=\frac{d}{d t}\left[\mathcal{J}\left(\Omega_{t}\right)\right]_{\mid t=0}=\Phi^{\prime}\left(j_{s r}(\Omega)\right) \frac{d}{d t}\left[j_{s r}\left(\Omega_{t}\right)\right]_{\mid t=0}+\ell \frac{d}{d t}\left[\left|\Omega_{t}\right|\right]_{\mid t=0} .
$$

Of course, this is only valid if the derivatives $\frac{d}{d t}\left[j_{s r}\left(\Omega_{t}\right)\right]_{\mid t=0}$ and $\frac{d}{d t}\left[\left|\Omega_{t}\right|\right]_{\mid t=0}$ exist. For this latter one this is obviously true. In the two dimensional case $N=2$ in which we henceforth place ourselves we have

$$
\frac{d}{d t}\left[\left|\Omega_{t}\right|\right]_{\mid t=0}=\left\{\begin{array}{lll}
-\pi & \text { if } & \hat{x} \in \Omega, \\
\pi & \text { if } & \hat{x} \in D \backslash \bar{\Omega} .
\end{array}\right.
$$

The expression of $\frac{d}{d t}\left[j_{s r}\left(\Omega_{t}\right)\right]_{\mid t=0}$ has been obtained in Theorem 5.3 upon Assumption 5.1. The operator $G_{\Omega}$ that satisfies Assumption 5.1 is associated with the topological derivative of the classical compliance. Its expression is known as (see $[4,6])$ :

$$
\left\langle G_{\Omega} \xi, \xi\right\rangle=-\pi \frac{r-1}{\kappa r+1} \frac{\kappa+1}{2}\left[2 \sigma: e+\frac{(\varepsilon-1)(\kappa-2)}{\kappa+2 \varepsilon-1} \operatorname{tr} \sigma \operatorname{tr} e\right]
$$


with

$$
r=\left\{\begin{array}{lll}
\varepsilon & \text { if } & \hat{x} \in \Omega, \\
\varepsilon^{-1} & \text { if } & \hat{x} \in D \backslash \bar{\Omega},
\end{array}\right.
$$

$\kappa=\left(\lambda_{L}+3 \mu_{L}\right) /\left(\lambda_{L}+\mu_{L}\right), \lambda_{L}, \mu_{L}$ the Lamé coefficients of the material, and $(\sigma, e)$ the stress and strain tensors respectively at point $\hat{x}$ for the load $B_{\Omega} \xi$.

A necessary optimality condition for this class of perturbations is clearly

$$
g_{\Omega}(\hat{x}) \geq 0 \quad \forall \hat{x} \in D,
$$

which is the starting point of our algorithm.

6.3. Description of the algorithm. In order to solve (6.1) we use the algorithm introduced in [6] and further analyzed in [5]. We recall its main features. Each domain $\Omega$ is represented by a smooth function $\psi_{\Omega}: D \rightarrow \mathbb{R}$ such that

$$
\Omega=\left\{x \in D, \psi_{\Omega}(x)<0\right\} .
$$

Such a level-set representation is very standard in shape optimization. It is usually combined with a Hamilton-Jacobi evolution equation, see $[3,31]$ to cite only the seminal works. However the Hamilton-Jacobi formulation does not allow to easily nucleate holes, which can be a serious limitation in topology optimization. We proceed differently, defining first the signed topological derivative as

$$
\tilde{g}_{\Omega}(\hat{x})=\left\{\begin{array}{rrr}
-g_{\Omega}(\hat{x}) & \text { if } & \hat{x} \in \Omega, \\
g_{\Omega}(\hat{x}) & \text { if } & \hat{x} \notin \Omega .
\end{array}\right.
$$

It appears that (6.1) will be solved as soon as

$$
\tilde{g}_{\Omega} \sim \psi_{\Omega}
$$

with the equivalence relation $\sim$ defined by

$$
\psi_{1} \sim \psi_{2} \Longleftrightarrow \exists \alpha>0, \psi_{1} \sim \alpha \psi_{2} .
$$

We apply to (6.2) the fixed point iteration with relaxation, i.e., the update of the function $\psi_{\Omega}$ at iteration $k$ is

$$
\psi_{\Omega_{k+1}} \sim\left(1-\omega_{k}\right) \psi_{\Omega_{k}}+\omega_{k} \tilde{g}_{\Omega_{k}} .
$$

The parameter $\omega_{k} \in(0,1]$ acts as step size and is fixed at every iteration by a line search.

Remark 6.1. Consider a combination of disjoint topological perturbations, such as for instance

$$
\Omega_{t}=\Omega \backslash \bigcup_{i=1}^{n} \bar{B}\left(\hat{x}_{i}, \rho(t)\right)
$$

The topological derivative of the compliance is additive with respect to the perturbation (see [7]), i.e.,

$$
G_{\Omega}=\sum_{i=1}^{n} G_{\Omega, i}
$$

where $G_{\Omega, i}$ is the topological derivative for a single perturbation. The topological derivative of $\Omega \rightarrow j_{s r}(\Omega)$ for the combination of perturbations is then

$$
\frac{d}{d t}\left[j_{s r}\left(\Omega_{t}\right)\right]_{\mid t=0}=\inf _{\bar{\xi} \in \Xi} \bar{\mu} \sum_{i=1}^{n}\left\langle G_{\Omega, i}\left(\xi^{N}+\bar{\xi}\right), \xi^{N}+\bar{\xi}\right\rangle \geq \sum_{i=1}^{n} \inf _{\bar{\xi} \in \Xi} \bar{\mu}\left\langle G_{\Omega, i}\left(\xi^{N}+\bar{\xi}\right), \xi^{N}+\bar{\xi}\right\rangle .
$$


This means that $j_{s r}$ is superadditive with respect to the perturbation, hence a combination of descent directions for the functional $\mathcal{J}$ still provides a descent direction (recall that $\Phi$ is decreasing).

For solving the dual problem (3.14) in the easy case and the hard case I, we have contented ourselves with the bisection method, as in our examples the space $\mathcal{H}$ was of small dimension.

Also, we have always encountered simple eigenvalues $\lambda_{\max }$. In such cases the set of critical loads $\Xi$ consists of at most two points, therefore solving the minimization problem in (5.1) is trivial. Further investigation would be needed to design a numerical procedure able to deal with the general case.

\section{Numerical examples}

In the subsequent computations, the linear elasticity equations are solved by means of $P 1$ finite elements (recall that the space dimension is $N=2$ ), leading to a stiffness matrix denoted by $K_{\Omega}$. We consider a finite-dimensional space of perturbations $\mathcal{H}$, say $\mathcal{H}=\mathbb{R}^{m}$ equipped with its canonical inner product and its canonical basis $\left(e_{i}\right)_{i=1}^{m}$. Each $e_{i}$ corresponds to an applied load $\phi_{i}$ and a force vector $F_{i}$ in the finite element framework. Then the matrix $Q_{\Omega}$ admits the entries:

$$
\left[Q_{\Omega}\right]_{i j}=\frac{1}{2} F_{i}^{T} K_{\Omega}^{-1} F_{j} .
$$

7.1. Beam. The hold all domain $D$ is the unit square $] 0,1[\times] 0,1[$, with a Dirichlet boundary condition on the left side. We denote by $p$ the middle of the right side and by $\phi_{1}$ the unit horizontal force applied at $p$. The nominal load $f_{\Omega}^{N}$ corresponds to $\phi_{1}$.

At first we consider a one-dimensional space $\mathcal{H}$, for which the value $\xi=1$ of the parameter corresponds to the force $\phi_{1}$. The threshold $\alpha$ is chosen as 10 times the compliance of the initial domain, which is the band $] 0,1[\times] 0.4,0.6[$, under the nominal load. The Lagrange multiplier for the area is fixed to $\ell=10$. In this situation, only the easy case occurs. The optimized domain is represented in Figure 1, left. The convergence history of the criterion $\mathcal{J}$ is shown in Figure 2, left.

Next we add a unit vertical force $\phi_{2}$, still applied at point $p$, and represented by the parameter $\xi=(0,1)$ in the space $\mathcal{H}=\mathbb{R}^{2}$. The force $\phi_{1}$, represented by the parameter $\xi^{N}=(1,0)$, remains the nominal load. The other data are unchanged. The optimized domain is given in Figure 1, right, with the convergence history in Figure 2, right. For comparison, note that the area of this domain (0.168) is close to the area obtained in the previous case $(0.160)$. The configuration at convergence is the hard case II, with the critical loads (including the nominal load) corresponding to the forces $1.09 \phi_{1} \pm 0.76 \phi_{2}$.

7.2. Mast. For this problem the hold-all domain $D$ is the union of the rectangles ]$-1,1[\times] 0,4[$ and $]-2,2[\times] 4,6[$. A Dirichlet boundary condition is applied at the bottom side. We consider 4 forces:

- $\phi_{1}$ is the force of components $(0,-1)$ applied at point $p_{1}=(-1,4)$,

- $\phi_{2}$ is the force of components $(0,-1)$ applied at point $p_{2}=(1,4)$,

- $\phi_{3}$ is the force of components $(1,0)$ applied at point $p_{1}$,

- $\phi_{4}$ is the force of components $(1,0)$ applied at point $p_{2}$. 

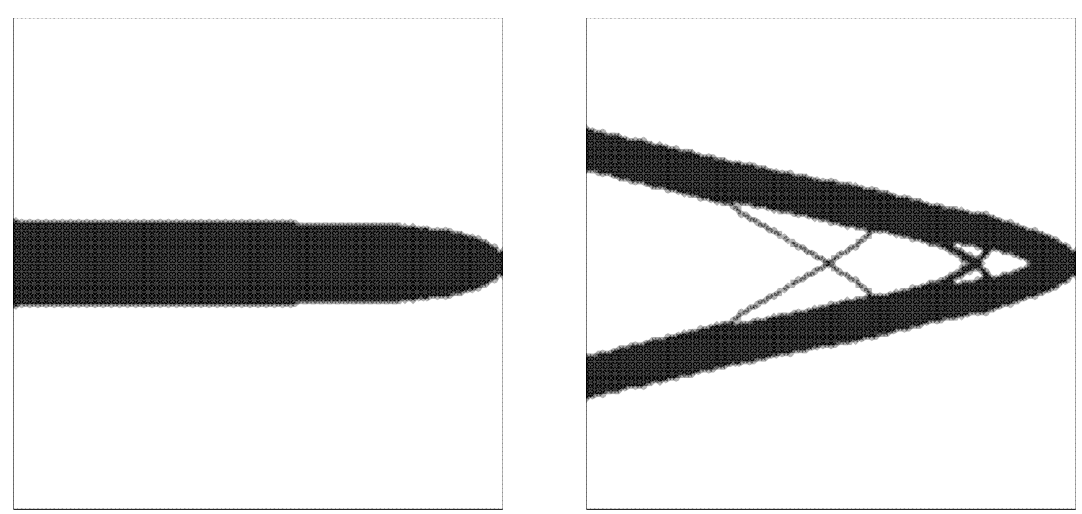

Figure 1. Beam: obtained domains for one load (left) and two loads (right)
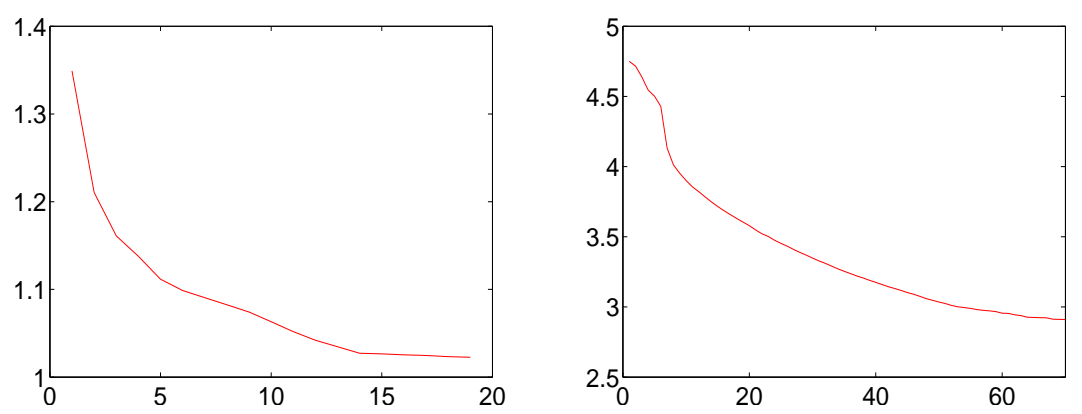

Figure 2. Beam: convergence histories for one load (left) and two loads (right)

The nominal load corresponds to the forces $\phi_{1}$ and $\phi_{2}$ applied simultaneously.

As first case we again consider a one-dimensional space of perturbations, spanned by the nominal load. The forces $\phi_{3}$ and $\phi_{4}$ are not taken into account. The initialization is the full domain $D$, and $\alpha$ is chosen as 10 times the compliance of this domain under the nominal load. The Lagrange multiplier $\ell$ is fixed to 0.8 . The optimized domain is represented in Figure 3, left.

Then we consider a two-dimensional space of perturbations, spanned by the forces $\phi_{1}$ and $\phi_{2}$ applied independently. All the other data are unchanged. The optimized domain is represented in Figure 3, middle. Due to the symmetry of the problem, this is a hard case II. The critical loads are $1.98 \phi_{1}+1.01 \phi_{2}$ and $1.01 \phi_{1}+1.98 \phi_{2}$.

Finally we consider four independent perturbations, given by the forces $\phi_{1}, \phi_{2}$, $\phi_{3}$ and $\phi_{4}$. We obtain the domain represented in Figure 3, right. This is again a hard case II, with the critical loads given by $1.28 \phi_{1}+1.09 \phi_{2}-0.30 \phi_{3}-0.39 \phi_{4}$ and its symmetric $1.09 \phi_{1}+1.28 \phi_{2}+0.39 \phi_{3}+0.30 \phi_{4}$.

$>$ From the first case to the last one, we clearly observe, first, a stiffening under non-symmetric vertical load, then, a stiffening under horizontal load. 


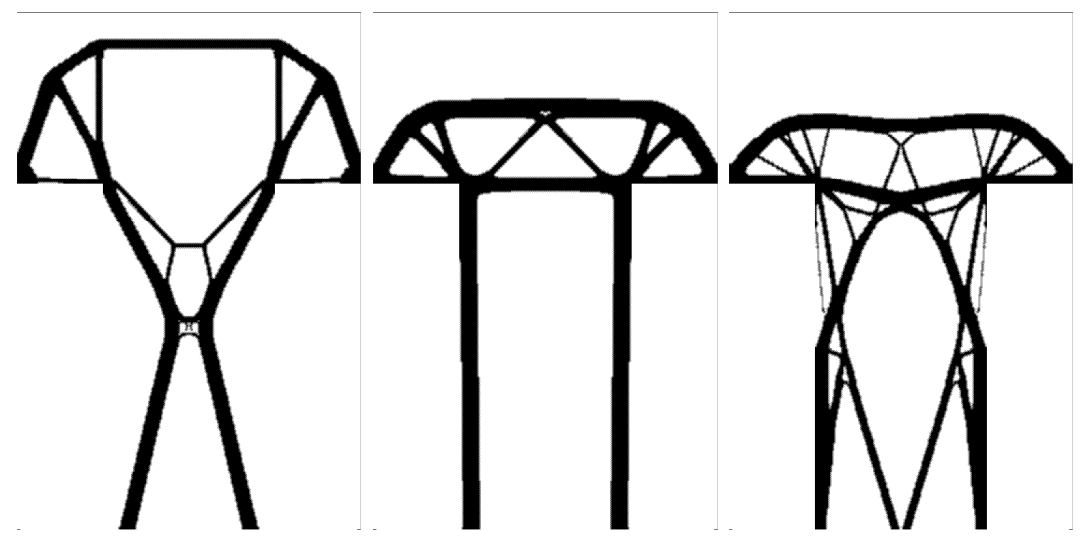

Figure 3. Mast: obtained domains for one load (left), two loads (middle) and four loads (right)

\section{Appendix A. LAGRANGIAN DUALiTy}

Here we gather useful results on general Lagrangian duality theory, which are essentially reformulations of classical results found in [26, 28, 33]. We nevertheless provide concise proofs for completeness.

Let $X, Y$ be two sets and $L: X \times Y \rightarrow \overline{\mathbb{R}}:=\mathbb{R} \cup\{-\infty,+\infty\}$ be an application, called the Lagrangian. We define

$$
\begin{array}{ll}
f(x)=\sup _{y \in Y} L(x, y), & x \in X, \\
g(y)=\inf _{x \in X} L(x, y), & y \in Y .
\end{array}
$$

The duality theory aims at finding relations between the primal problem

$$
\text { Minimize } f(x), \quad x \in X,
$$

and the so-called dual problem

$$
\text { Maximize } g(y), \quad y \in Y \text {. }
$$

We denote by $v(\mathscr{P})=\inf _{x \in X} f(x)$ and $v(\mathscr{D})=\sup _{y \in Y} g(y)$ the values of the primal and the dual problems, respectively.

Moreover, for any $y \in Y$, we consider the problem

$\left(\mathscr{L}_{y}\right) \quad$ Minimize $L(x, y), \quad x \in X$,

with value $v\left(\mathscr{L}_{y}\right)=\inf _{x \in X} L(x, y)=g(y)$. We always have (weak duality):

$$
\forall y \in Y, \quad v\left(\mathscr{L}_{y}\right) \leq v(\mathscr{D}) \leq v(\mathscr{P}) .
$$

Theorem A.1. (1) For all $y \in Y$ it holds

$$
\operatorname{argmin}\left(\mathscr{L}_{y}\right) \cap[L(., y)=f] \subset \operatorname{argmin}(\mathscr{P}) .
$$

(2) For all $y \in Y$ it holds

$$
v(\mathscr{P})=v\left(\mathscr{L}_{y}\right) \Longleftrightarrow\left\{\begin{array}{l}
y \in \operatorname{argmax}(\mathscr{D}), \\
v(\mathscr{D})=v(\mathscr{P}) .
\end{array}\right.
$$


(3) We have the relations:

$$
v(\mathscr{P})=v\left(\mathscr{L}_{y}\right) \Longrightarrow \operatorname{argmin}\left(\mathscr{L}_{y}\right) \cap[L(., y)=f]=\operatorname{argmin}(\mathscr{P}) .
$$

(4) For all $y \in Y$ it holds

$$
\operatorname{argmin}\left(\mathscr{L}_{y}\right) \cap[L(., y)=f] \neq \emptyset \Longrightarrow v\left(\mathscr{L}_{y}\right)=v(\mathscr{P})
$$

hence

(A.6) $\operatorname{argmin}\left(\mathscr{L}_{y}\right) \cap[L(., y)=f] \neq \emptyset \Longrightarrow \operatorname{argmin}\left(\mathscr{L}_{y}\right) \cap[L(., y)=f]=\operatorname{argmin}(\mathscr{P})$.

(5) We always have

$$
\left\{y \in Y \mid \operatorname{argmin}\left(\mathscr{L}_{y}\right) \cap[L(., y)=f] \neq \emptyset\right\} \subset \operatorname{argmax}(\mathscr{D}) .
$$

Proof. (1) If $\bar{x} \in \operatorname{argmin}\left(\mathscr{L}_{y}\right) \cap[L(., y)=f]$ then, for all $x \in X, f(\bar{x})=$ $L(\bar{x}, y) \leq L(x, y) \leq f(x)$.

(2) If $v\left(\mathscr{L}_{y}\right)=v(\mathscr{P})$, using (A.1), one obtains $v(\mathscr{D})=v(\mathscr{P})=v\left(\mathscr{L}_{y}\right)=g(y)$ and $y \in \operatorname{argmax}(\mathscr{D})$. If $y \in \operatorname{argmax}(\mathscr{D})$ and $v(\mathscr{D})=v(\mathscr{P})$ then $v\left(\mathscr{L}_{y}\right)=$ $g(y)=v(\mathscr{D})=v(\mathscr{P})$.

(3) If $\bar{x} \in \operatorname{argmin}(\mathscr{P})$ then $L(\bar{x}, y) \leq f(\bar{x})=v(\mathscr{P})=v\left(\mathscr{L}_{y}\right) \leq L(x, y)$ for all $x \in X$. In particular $L(\bar{x}, y)=f(\bar{x})$ and $\bar{x} \in \operatorname{argmin}\left(\mathscr{L}_{y}\right)$. The reverse inclusion is given by (A.2).

(4) If $\bar{x} \in \operatorname{argmin}\left(\mathscr{L}_{y}\right) \cap[L(., y)=f]$ then

$$
v(\mathscr{P})=\inf _{x \in X} f(x) \leq f(\bar{x})=L(\bar{x}, y)=v\left(\mathscr{L}_{y}\right)=\inf _{x \in X} L(x, y) \leq \inf _{x \in X} f(x)=v(\mathscr{P}) .
$$

(5) If $\operatorname{argmin}\left(\mathscr{L}_{y}\right) \cap[L(., y)=f] \neq \emptyset$, then using (A.5), one infers $v\left(\mathscr{L}_{y}\right)=$ $v(\mathscr{P})$. We conclude using (A.3).

Acknowledgements. The authors thank the anonymous referees for their careful reading, useful comments and suggestions.

\section{REFERENCES}

[1] G. Allaire. Conception optimale de structures, volume 58 of Mathématiques \&s Applications (Berlin) [Mathematics 83 Applications]. Springer-Verlag, Berlin, 2007. With the collaboration of Marc Schoenauer (INRIA) in the writing of Chapter 8.

[2] G. Allaire and C. Dapogny. A linearized approach to worst-case design in parametric and geometric shape optimization. Mathematical Models and Methods in Applied Sciences, 24(11):2199-2257, 2014.

[3] G. Allaire, F. Jouve, and A.-M. Toader. Structural optimization using sensitivity analysis and a level-set method. J. Comput. Phys., 194(1):363-393, 2004.

[4] S. Amstutz. Sensitivity analysis with respect to a local perturbation of the material property. Asymptot. Anal., 49(1-2):87-108, 2006.

[5] S. Amstutz. Analysis of a level set method for topology optimization. Optim. Methods Softw., 26(4-5):555-573, 2011.

[6] S. Amstutz and H. Andrä. A new algorithm for topology optimization using a level-set method. J. Comput. Phys., 216(2):573-588, 2006.

[7] S. Amstutz and M. Ciligot-Travain. Optimality conditions for shape and topology optimization subject to a cone constraint. SIAM J. Control Optim., 48(6):4056-4077, 2010.

[8] I. Babuška, F. Nobile, and R. Tempone. Worst case scenario analysis for elliptic problems with uncertainty. Numer. Math., 101(2):185-219, 2005.

[9] Y. Ben-Haim. Information-gap decision theory. Series on Decision and Risk. Academic Press Inc., San Diego, CA, 2001. Decisions under severe uncertainty.

[10] A. Ben-Tal, L. El Ghaoui, and A. Nemirovski. Robust optimization. Princeton Series in Applied Mathematics. Princeton University Press, Princeton, NJ, 2009. 
[11] M. P. Bendsøe and O. Sigmund. Topology optimization. Springer-Verlag, Berlin, 2003. Theory, methods and applications.

[12] J. F. Bonnans and A. Shapiro. Perturbation analysis of optimization problems. Springer Series in Operations Research. Springer-Verlag, New York, 2000.

[13] A. Cherkaev and E. Cherkaev. Optimal design for uncertain loading condition. In Homogenization, volume 50 of Ser. Adv. Math. Appl. Sci., pages 193-213. World Sci. Publ., River Edge, NJ, 1999.

[14] E. Cherkaev and A. Cherkaev. Principal compliance and robust optimal design. J. Elasticity, 72(1-3):71-98, 2003. Essays and papers dedicated to the memory of Clifford Ambrose Truesdell III. Vol. III.

[15] F. de Gournay, G. Allaire, and F. Jouve. Shape and topology optimization of the robust compliance via the level set method. ESAIM Control Optim. Calc. Var., 14(1):43-70, 2008.

[16] J.-M. Feng, G.-X. Lin, R.-L. Sheu, and Y. Xia. Duality and solutions for quadratic programming over single non-homogeneous quadratic constraint. J. Global Optim., 54(2):275-293, 2012.

[17] O. Flippo and B. Jansen. Duality and sensitivity in nonconvex quadratic optimization over an ellipsoid. European Journal of Operational Research, 94(1):167-178, 1996.

[18] C. Fortin and H. Wolkowicz. The trust region subproblem and semidefinite programming. Optim. Methods Softw., 19(1):41-67, 2004.

[19] A. L. Fradkov and V. A. Jakubovič. The $S$-procedure and the duality relation in convex quadratic programming problems. Vestnik Leningrad. Univ., (1 Mat. Meh. Astronom. Vyp. 1):81-87, 155, 1973.

[20] A. L. Fradkov and V. A. Jakubovič. The $S$-procedure and the duality relation in convex quadratic programming problems. Vestnik Leningrad. Univ., (Vol. 6, n ${ }^{\circ}$ 2):101-109, 1979.

[21] S. Garreau, P. Guillaume, and M. Masmoudi. The topological asymptotic for PDE systems: the elasticity case. SIAM J. Control Optim., 39(6):1756-1778 (electronic), 2001.

[22] D. M. Gay. Computing optimal locally constrained steps. SIAM J. Sci. Statist. Comput., 2(2):186-197, 1981.

[23] X. Guo, W. Zhang, and L. Zhang. Robust structural topology optimization considering boundary uncertainties. Comput. Methods Appl. Mech. Engrg., 253:356-368, 2013.

[24] D. Hinrichsen and A. J. Pritchard. Real and complex stability radii: a survey. In Control of uncertain systems (Bremen, 1989), volume 6 of Progr. Systems Control Theory, pages 119-162. Birkhäuser Boston, Boston, MA, 1990

[25] D. Hinrichsen, A. J. Pritchard, and S. B. Townley. Riccati equation approach to maximizing the complex stability radius by state feedback. Internat. J. Control, 52(4):769-794, 1990.

[26] J.-B. Hiriart-Urruty and C. Lemaréchal. Convex analysis and minimization algorithms. II, volume 306 of Grundlehren der Mathematischen Wissenschaften [Fundamental Principles of Mathematical Sciences]. Springer-Verlag, Berlin, 1993. Advanced theory and bundle methods.

[27] A. D. Ioffe and V. M. Tihomirov. Theory of extremal problems, volume 6 of Studies in Mathematics and its Applications. North-Holland Publishing Co., Amsterdam-New York, 1979. Translated from the Russian by Karol Makowski.

[28] C. Lemaréchal. The omnipresence of Lagrange. Ann. Oper. Res., 153:9-27, 2007.

[29] J. J. More. Generalizations of the trust region problem. Optimization Methods and Software, 2(3-4):189-209, 1993.

[30] A. A. Novotny and J. Sokołowski. Topological derivatives in shape optimization. Interaction of Mechanics and Mathematics. Springer, Heidelberg, 2013.

[31] S. Osher and J. A. Sethian. Fronts propagating with curvature-dependent speed: algorithms based on Hamilton-Jacobi formulations. J. Comput. Phys., 79(1):12-49, 1988.

[32] F. Rendl and H. Wolkowicz. A semidefinite framework for trust region subproblems with applications to large scale minimization. Math. Programming, 77(2, Ser. B):273-299, 1997. Semidefinite programming.

[33] R. T. Rockafellar. Conjugate duality and optimization. Society for Industrial and Applied Mathematics, Philadelphia, Pa., 1974. Lectures given at the Johns Hopkins University, Baltimore, Md., June, 1973, Conference Board of the Mathematical Sciences Regional Conference Series in Applied Mathematics, No. 16.

[34] J. Sokołowski and A. Żochowski. On the topological derivative in shape optimization. SIAM J. Control Optim., 37(4):1251-1272 (electronic), 1999. 
[35] D. C. Sorensen. Newton's method with a model trust region modification. SIAM J. Numer. Anal., 19(2):409-426, 1982.

[36] R. J. Stern and H. Wolkowicz. Trust region problems and nonsymmetric eigenvalue perturbations. SIAM J. Matrix Anal. Appl., 15(3):755-778, 1994.

[37] R. J. Stern and H. Wolkowicz. Indefinite trust region subproblems and nonsymmetric eigenvalue perturbations. SIAM J. Optim., 5(2):286-313, 1995.

Laboratoire de Mathématiques d'Avignon, Faculté des Sciences, 33 rue Pasteur, 84000 Avignon, France.

Laboratoire de Mathématiques d’Avignon, Faculté des Sciences, 33 rue Pasteur, 84000 Avignon, France. 